\title{
Clustering of Protein Structural Fragments Reveals Modular Building Block Approach of Nature
}

\author{
Ashish V. Tendulkar ${ }^{1}$, Anand A. Joshi ${ }^{2}$, Milind A. Sohoni ${ }^{3}$ and \\ Pramod P. Wangikar ${ }^{2 *}$
}

\author{
${ }^{1}$ Kanwal Rekhi School of \\ Information Technology, Indian \\ Institute of Technology \\ Bombay, Powai \\ Mumbai 400 076, India
}

${ }^{2}$ Department of Chemical

Engineering, Indian Institute of

Technology, Bombay, Powai

Mumbai 400 076, India

${ }^{3}$ Department of Computer Science and Engineering Indian Institute of Technology Bombay, Powai

Mumbai 400 076, India

\begin{abstract}
Structures of peptide fragments drawn from a protein can potentially occupy a vast conformational continuum. We co-ordinatize this conformational space with the help of geometric invariants and demonstrate that the peptide conformations of the currently available protein structures are heavily biased in favor of a finite number of conformational types or structural building blocks. This is achieved by representing a peptides' backbone structure with geometric invariants and then clustering peptides based on closeness of the geometric invariants. This results in 12,903 clusters, of which 2207 are made up of peptides drawn from functionally and/or structurally related proteins. These are termed "functional" clusters and provide clues about potential functional sites. The rest of the clusters, including the largest few, are made up of peptides drawn from unrelated proteins and are termed "structural" clusters. The largest clusters are of regular secondary structures such as helices and beta strands as well as of beta hairpins. Several categories of helices and strands are discovered based on geometric differences. In addition to the known classes of loops, we discover several new classes, which will be useful in protein structure modeling. Our algorithm does not require assignment of secondary structure and, therefore, overcomes the limitations in loop classification due to ambiguity in secondary structure assignment at loop boundaries.
\end{abstract}

Keywords: geometric invariants; protein structure comparison; secondary structure; loop

\section{Introduction}

Globular proteins are made up of regular secondary structures such as $\alpha$-helices and $\beta$-strands and non-regular secondary structures, which are referred to as loops. The regular secondary structures are defined based on a regular pattern of the backbone torsion angles for consecutive amino acid residues or periodic patterns of hydrogen bonding between the backbone $\mathrm{NH}$ and $\mathrm{C}=\mathrm{O}$ groups. ${ }^{1}$ Loops are the regions that join the regular secondary structures and lack the regularity of torsion angles for consecutive residues. In fact, loops, polypeptide fragments from proteins, which trace a "loop-shaped" path in three-dimensional space, were considered to be random coils until recently. ${ }^{2}$ Loops are highly compact substructures and are typically situated at the protein surface. Certain loop geometries have been shown to recur across non-homologous proteins, which forms the basis for classification of loops into structural families. For example, the $\beta$-hairpin and $\alpha$-turn families of loops have been described in detail. ${ }^{1,3-7}$ A much wider classification of loops of various categories has been provided by Kwasigroch et al. ${ }^{8}$ and by Oliva et al. ${ }^{9}$ Loop classification has important implications in protein structure modeling and in fitting the NMR distance constraints or an X-ray crystallography electron density map to a loop geometry. On the contrary, no such fine-grained classification has been attempted for $\alpha$-helices and $\beta$-strands, although detectable variations are reported in these regular secondary structures. ${ }^{10}$

Several authors have considered loops as 
connections between specific secondary structures, rather than as independent structural motifs. ${ }^{8,9,11}$ Thus, loop classification begins with assignment of "frontier" amino acid residues to the secondary structures that a loop connects. The loops are first classified based on the types of secondary structures that they connect followed by the number of residues in the loop and finally based on the loop geometry. Bystroff \& Baker ${ }^{12}$ have developed a library of sequence-structure motifs via clustering of the $k$-mer words derived from the amino acid sequences of the Protein Data Bank. The resultant clusters are further processed and pruned to obtain structural families of the $k$-mer peptide fragments. The library is likely to miss the structural motifs that do not correlate well with a sequence motif. Despite this limitation, the library has been shown to be useful in predicting the structure of proteins based on the sequence-structure patterns. ${ }^{13}$

It is now known that many families of proteins have evolved to perform multiple related functions with variations in loop regions on a relatively conserved framework. ${ }^{14}$ The immunoglobulin family is an excellent example, with the well-known hypervariable loop regions. Nature has evolved a system designed to bind virtually any antigen by variation in the sequence and length of just six loops. Despite the wide variation in the nature of antigen, loops in the immunoglobulin family have been classified into structural families via automated $^{14}$ or manual classification procedures to define canonical classes. ${ }^{15,16}$

Sequence signatures ${ }^{17}$ are a well-characterized feature of proteins. For example, the PROSITE, ${ }^{18}$ PRINTS $^{19}$ and Blocks ${ }^{20}$ databases provide an extensive survey of family/sub-family-wise sequence signatures based on multiple sequence alignments. While the PROSITE patterns are defined in principle as the shortest discriminating sequences, in order to improve the recognition power for distant relatives, multiple sequence motifs for a family have been introduced. These sequence patterns are considered to include those residues that play important roles in the function of the protein or in the formation of the core structure of the protein. Therefore, it is expected that for each conserved sequence pattern there is a common threedimensional structure, which can be considered characteristic of the function. As a result, some of these sequence signatures have been converted to structural signatures by superimposing the corresponding sub-structures in an attempt to define a three-dimensional functional pattern. ${ }^{21}$ Typically, each sequence signature covers only a family of homologous proteins. Thus, the structural templates derived from the PROSITE sequence signatures are not likely to include such structural motifs, which span across several protein families. Moreover, no information has been presented on the uniqueness of the structural motifs to a homologous family of the proteins.

We present a fine-grained classification of all types of regular and non-regular secondary struc- tures present in the current version of the PDB_95 protein structure data set. ${ }^{22}$ The input consisted of ca 1.2 million octapeptide structural fragments drawn from the PDB_95 set. Our method clusters the peptide fragments based purely on backbone structural similarity. We use geometric invariants to arrive at superimposability of structural fragments. We have previously demonstrated the utility of geometric invariants in clustering geometrically similar patterns of three to six amino acid residues. ${ }^{23}$ This eliminates the computationally explosive process of finding out the superposing transformation for all the possible pairs of fragments. The results provide a finer classification of the known secondary structural categories of $\alpha$-helices and $\beta$-strands in addition to providing thousands of new categories of nonregular secondary structures. For example, the $\beta$-strand fragments get classified into several clusters where the peptides from two different clusters are not superimposable. Several other clusters were of loops with or without the adjoining secondary structural elements and spanned across many SCOP superfamilies. Although the proteins as well as the fragments of these clusters were highly non-homologous, some key positions were highly conserved in the turn regions. In addition, several SCOP superfamily specific clusters were obtained.

\section{Algorithm}

In this section, we describe the algorithm for detection of recurring structural motifs from the protein universe (Figure 1). Here, the backbone structure of a peptide segment from a protein is referred to as structural motif. We use only the $C^{\alpha}$ atoms as an approximate representation of the backbone structure of a peptide. Our input dataset is comprised of PDB structures from the PDB_95 non-redundant set from the ASTRAL database ${ }^{22}$ where no two peptide chains are greater than 95\% identical. Our analysis considers overlapping peptide segments of $k$ amino acids.

\section{Geometric invariant theory}

Two peptides, $X$ and $Y$, are said to be superimposable if there exists a rigid body transformation (a group of translations and rotations), which brings the corresponding atoms in the two peptides close to each other. We denote this as $X \sim Y$ with $\sim$ being the equivalence relationship. Given a space $C$ of conformation of peptides, a real valued function $f: C \rightarrow \mathbb{R}$ is called an invariant if $X \sim Y$ implies $f(X) \approx f(Y)$. Examples of geometric invariants include volume, surface area and perimeter of a polyhedron. Previously, we have described the utility of geometric invariants in detecting recurring structural patterns in the protein universe. ${ }^{23}$ It is important to note that it is possible to determine the validity of $X \sim Y$ via 


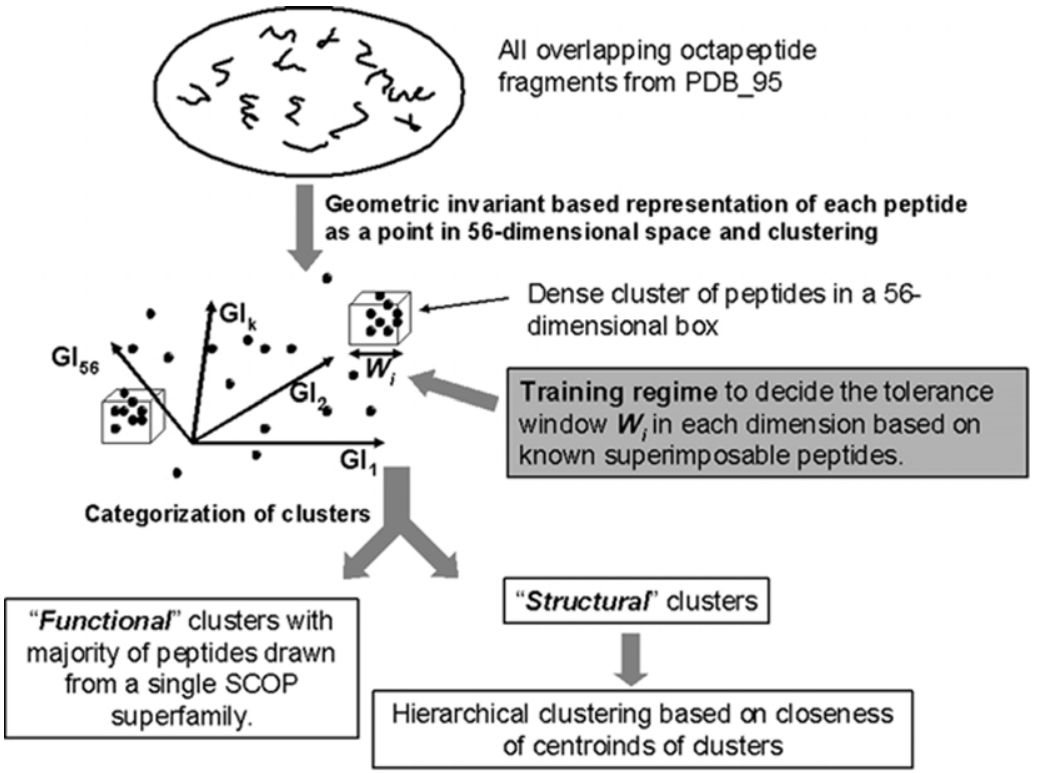

Figure 1. The overall algorithm for clustering of peptide fragment structures based on geometric invariants. geometric invariants without actually computing the transformation that superposes $X$ with $Y{ }^{24-26}$ The crucial point now is to be able to select a suite of invariants $F(X)=\left(f_{1}(X), f_{2}(X), \ldots, f_{k}(X)\right)$ that we can use to co-ordinatize the configuration space $C$. In some norm on $\mathbb{R}^{k}$, if $\|(X)-(Y)\|<\varepsilon$, then we declare $X$ and $Y$ to be superimposable.

\section{Representation and parameterization of a peptide segment structure}

We use the $\mathrm{C}^{\alpha}$ atoms to represent the backbone structure of a protein. Thus, a peptide structural fragment derived from a protein, say an octapeptide, is essentially a geometric structure with eight points (the eight $C^{\alpha}$ atoms) in $\mathbb{R}^{3}$ space with labels $C^{\alpha 1}, C^{\alpha 2}, \ldots, C^{\alpha 8}$ assigned to each of the points (Figure 2). We now construct several tetrahedra from these eight points. For example, the first tetrahedron is made up of points $C^{\alpha 1}, C^{\alpha 2}, C^{\alpha 3}$ and $\mathrm{C}^{\alpha 4}$. Likewise, we construct four more tetrahedra for an octapeptide with each tetrahedron having four consecutive points. We call these tetrahedra_gap_0. In addition, we construct tetrahedra with alternate points such as $C^{\alpha 1}, C^{\alpha 3}, C^{\alpha 5}$ and $C^{\alpha 7}$, which we label as tetrahedron_gap_1. It may be noted that the tetrahedra constructed here are a subset of all possible tetrahedra $\left({ }^{8} \mathrm{C}_{4}\right)$ that can be constructed from an octapeptide. For our purpose, we select a subset of these tetrahedra. For each tetrahedron, we compute several geometric invariants such as the perimeter, sum of edge squares, sum of centroid to point distances, etc. (Table 1). The signed volume of the tetrahedron is an important invariant as the sign can distinguish between the non-superimposable mirror images. The suite of invariants was carefully chosen after extensive trial and error on training data to address the following two issues: (1) for superimposable peptides, the invariants must be similar within a

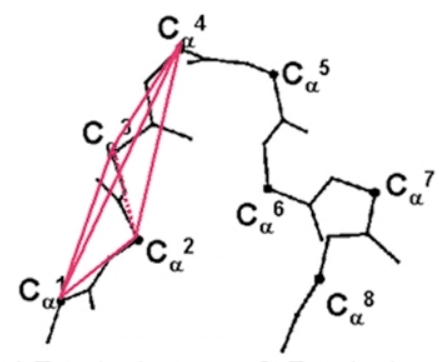

a) Tetrahedron gap 0: Tetrahedron constructed from consecutive $\mathrm{C}_{\alpha}$ atoms.

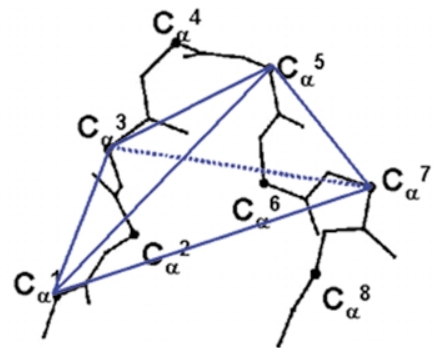

b) Tetrahedron_gap_1: Tetrahedron constructed from alternate $C_{\alpha}$ atoms

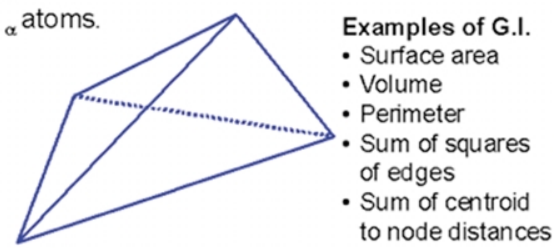

c) Geometric invariants associated with a tetrahedron
Figure 2. Construction of geometric objects from peptide structure and computation of geometric invariants. (a) Construction of tetrahedron_gap_0 with consecutive $C^{\alpha}$ atoms from an octapeptide. (b) Construction of tetrahedron_gap_1 with alternate $\mathrm{C}^{\alpha}$ atoms from an octapeptide. (c) Examples of geometric invariants associated with a tetrahedron. 
Table 1. The geometric invariants and the respective windows used in the clustering of peptide structural fragments

\begin{tabular}{lcc}
\hline & & Windows for \\
\cline { 2 - 3 } Geometric invariant and unit & Tetrahedron_gap_0 & Tetrahedron_gap_1 \\
\hline$\sqrt[3]{\text { Volume }(\AA)}$ & 1.7 & 2.8 \\
Perimeter $(\AA)$ & 2 & 4 \\
Sum of variances $(\AA)$ & 0.8 & 2 \\
$\sum\left(\right.$ Length $^{2}\left(\AA^{\circ}\right)$ & 35 & 110 \\
$\sum_{i \neq j}\left(\right.$ Length $_{i} \times$ Length $\left._{j}\right)\left(\AA^{2}\right)$ & 80 & 230 \\
$\left.\sum_{\text {Variance }}\right)^{2}\left(\AA^{2}\right)$ & 7 & 30 \\
$\sum_{i \neq j}\left(\right.$ Variance $_{i} \times$ Variance $\left._{j}\right)\left(\AA^{2}\right)$ & 1.5 & 27 \\
Distance $_{i j}(j=i+3)(\AA)$ & - & - \\
Distance $_{i j}(j=i+6)(\AA)$ & & 1.75 \\
\hline
\end{tabular}

tolerance limit; and (2) for a pair of nonsuperimposable peptides $X \nsim Y$, there must be at least one geometric invariant such that $f(X) \nsim f(Y)$.

For each octapeptide segment (including the overlapping ones), the corresponding tetrahedra are generated. This includes two tetrahedra_gap_1 and five tetrahedra_gap_0. For each tetrahedron, eight geometric invariants are calculated (Figure 2). Thus, an octapeptide is described by a vector of 56 geometric invariants. In a similar representation, a longer peptide, say a decapeptide, is represented by a larger number of invariants. In this analysis, it is imperative that a given tetrahedron may be the third tetrahedron of one peptide and the first tetrahedron of another peptide. To avoid duplication, the string of valid tetrahedra and their invariants is listed for each protein. For peptides, the relevant tetrahedra and their invariants are called from this list to map peptides into vectors.

\section{Clustering algorithm}

The clustering algorithm is a standard data mining subroutine and its implementation is a modified version of that described by us earlier. ${ }^{23}$ The input is a collection of points in $k$-dimensional space, which are essentially the collection of peptide segment structures described by the $k$ geometric invariants. The clustering algorithm searches for dense regions in this $k$-dimensional space. A region is defined as the $k$-dimensional box obtained by the conjunction of the tolerance windows in each dimension. The search for dense regions is carried out in a breadth-first manner as described before ${ }^{23}$ such that all the peptides in the protein universe are first passed to the first dimension to create clusters that satisfy the tolerance criterion on the first dimension followed by the second dimension and so on.

At this point, we have introduced an implementation trick as follows. The 56 invariants describing an octapeptide are drawn from seven different tetrahedra as described above. A given tetrahedron $i$ could be the third tetrahedron for peptide $i-2$ or the first tetrahedron for peptide $i$. The 56-dimensional representation of octapeptides may lead to redundancy in data and execution of the clustering algorithm. Thus, we first cluster the two types of tetrahedra, tetrahedra_gap_1 and tetrahedra_gap_2, each on an eight-dimensional space, separately. The search for clusters of octapeptides is now a true search problem as follows. A cluster of octapeptides with indices $i(i=K, L, \ldots, N)$ is a valid cluster if there exist two clusters of tetrahedron_gap_1, one with indices $g(g=K, L, \ldots, N)$ and one with indices $h(h=K+1, L+1, \ldots, N+1)$, and five clusters of tetrahedron_gap_0, one with indices $j(j=K, L, \ldots, N)$, one with indices $k \quad(k=K+1, L+1, \ldots, N+1)$, one with indices $l(l=K+2, L+2, \ldots, N+2)$, one with indices $m(m=$ $K+3, L+3, \ldots, N+3)$ and one with indices $n(n=$ $K+4, L+4, \ldots, N+4)$. These could be subsets of the detected clusters of the tetrahedra. The search operation is easily carried out via repetitive set intersection operations. Clusters of longer peptides, say decapeptides, can be obtained by applying conjunctive criteria of additional tetrahedra as a larger number of tetrahedra are contained within a decapeptide compared to an octapeptide. Moreover, additional types of tetrahedra, such as tetrahedra_gap_2 are introduced while examining the clusters of longer peptide segments.

The tolerance windows are obtained from a training regime as described earlier. ${ }^{23}$ We select a collection of peptide segment structures, which are known to be superimposable with each other. For each invariant, a tolerance value, Window $\left(W_{i}\right)$, is chosen that exceeds the variance in the peptides in the training data. The second parameter, the threshold number of peptides required to declare a region as dense, was selected to be 5 after initial trial runs on the PDB_95 dataset. This allows us to capture the family-specific structural motifs as well as loops in addition to the standard secondary structural classes.

\section{Post-processing of clusters}

The clustering algorithm is likely to generate 
overlapping clusters, which may be adjacent in the $k$-dimensional space of geometric invariants. Such adjacent clusters were merged if the centroids of the two clusters satisfied the conjunctive criteria of closeness on all the $k$ coordinates. The closeness criteria for the centroids of clusters $l$ and $m$ was set as $\left|X_{i}^{l}-X_{i}^{m}\right|<0.3 x W_{i}$, where $W_{i}$ is the maximum allowable width of the cluster in the $i$ th dimension.

\section{Estimation of sequence conservation-based information content and average phi-psi angles of peptides in a cluster}

Information content based on amino acid conservation pattern was estimated for each of the eight positions for each cluster and then sequence logos were constructed as described. ${ }^{27} \mathrm{~A}$ regular expression was derived for each position to indicate the preferred amino acids or excluded amino acids at that position in a cluster. The backbone torsion angles (phi-psi angles) were calculated by considering the co-ordinates of $\mathrm{N}$, $\mathrm{C}^{\alpha}$, and $\mathrm{C}$ atoms of the each of the amino acid residues of the octapeptide with the $C$ atom of the residue preceding the octapeptide and $\mathrm{N}$ atom of the residue subsequent to the octapeptide. The average torsion angle (phi or psi) at a given residue position in a cluster of superimposable peptides were obtained as an arithmetic mean of the torsion angles calculated for each peptide separately.

\section{Hierarchical clustering of clusters}

Each cluster of peptides is represented as a centroid or a point in the 56-dimensional space. These clusters are further clustered via a hierarchical clustering algorithm. The hierarchical tree is divided into 30 supergroups at major branch points while each supergroup is divided into up to 19 groups. This provides a clear relationship between the clusters.

\section{Results}

The input dataset consisted of the PDB structures $^{28,29}$ of 4849 peptide chains, with a cumulative chain length of 1,245,745 amino acid residues, drawn from the PDB_95 dataset of the ASTRAL database. ${ }^{28}$ Approximately 1,189,057 valid overlapping opctapeptide structural fragments were drawn from these peptide chains. Each octapeptide was represented as a vector of 56 geometric invariants. Thus, each peptide is a point in the 56-dimensional space. A clustering algorithm was employed to group peptides based on closeness of geometric invariants. A deterministic clustering algorithm was used where the maximum allowable width in the $i$ th dimension, $W_{i}$, was predecided (Table 1) using training data. The training data consisted of true structural matches for structural signatures derived from PROSITE signatures and were adapted from reported results. ${ }^{21}$ The cutoff for minimum size of the clusters was set at five peptides and was independent of the number of proteins from which the peptides are drawn. Clustering and post-processing of the clusters resulted in 12,903 clusters of peptide fragment structures. The PDB structures used in the input and the details of each cluster are provided as Supplementary Material. These clusters together account for 466,030 octapeptides and cover 956,823 amino acid residues out of the 1,245,745 residues in the input protein structure data. Of the 12,903 clusters, the largest 100 clusters account for 261,342 peptides and cover 536,197 amino acid residues. To ascertain that the peptides in a cluster are indeed superimposable, five randomly chosen peptides from each cluster were superimposed using SwissPdbViewer. ${ }^{30}$ The RMSD values for the superposed peptides were below $1.0 \AA$ for each cluster.

\section{(A) Distribution of clusters By Information Content}

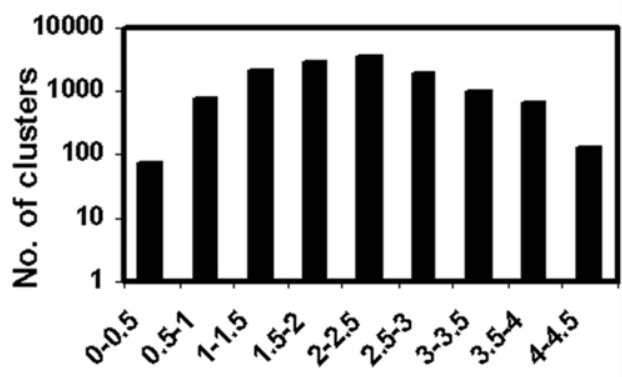

Avg. information content of the cluster

\section{(B) Distribution of clusters By Cluster size.}

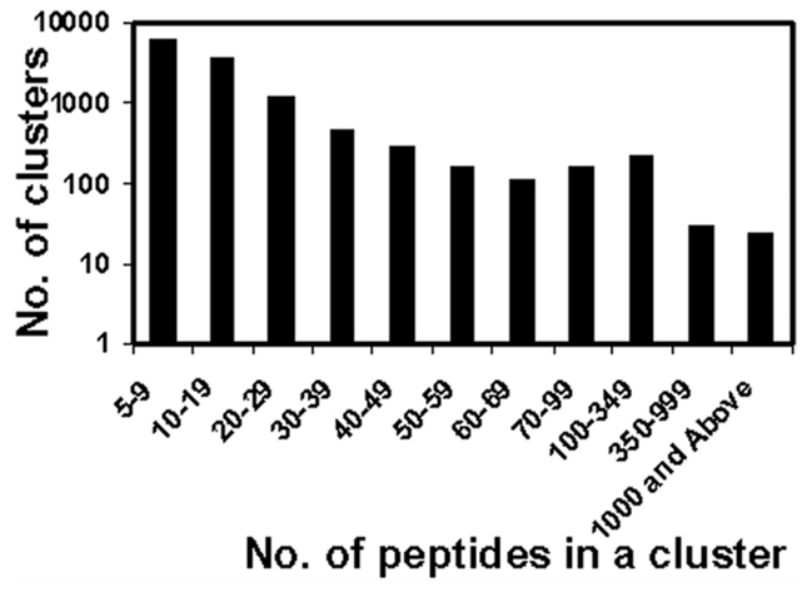

Figure 3. (A) Distribution of information content. (B) Distribution of cluster size. 


\section{Categorization of clusters}

It was of interest to determine whether the peptides of a cluster are drawn from proteins that have overall structural/sequence homology with each other. SCOP classification provides a hierarchical classification of PDB structures. ${ }^{31}$ PDB structures of a common SCOP superfamily are believed to share functional and/or structural relationship with each other. Thus, we tagged our clusters as "functional" clusters if more than 70\% of the peptides of that cluster belong to a common SCOP superfamily. All other clusters were termed as "structural" clusters as the structural similarity of the peptide fragments of these clusters is not based on evolutionary/functional relationships between proteins. A total of 131 superfamilies were found to have at least one functional cluster per family. Functional clusters were mainly made up of loop regions. The structural clusters were of regular secondary structures as well as of loops with or without the adjoining secondary structures.

A distribution of the cluster size is shown in
Figure 3A. The largest cluster consisted of 166,662 peptides followed by second largest cluster of 8257 peptides. We detected a total of 23 clusters with each cluster containing more than 1000 peptides. The large clusters typically tend to be structural clusters (Tables 2-4). The functional clusters tend to be small with the largest functional cluster of 306 peptides which was of the immunoglubulin superfamily.

A sequence conservation pattern for the octapeptides of each cluster was quantified in the form of information content for each residue position. ${ }^{27}$ Some of the clusters showed high residue conservation at one or more of the eight positions. The distribution of average information content of the clusters is shown in Figure 3B. The average of the information content of the eight positions reflects the overall sequence conservation of the octapeptides of the cluster. This information content is not related to the sequence alignment of the amino acid sequences of the proteins from which the peptides are drawn. The functional clusters show a consistently high information

Table 2. Geometric variations in regular secondary structures: summary of some representative "structural" clusters indicating variations in regular secondary structures

\begin{tabular}{|c|c|c|c|c|c|c|}
\hline ClusterID $^{\mathbf{a}}$ & $\begin{array}{l}\text { No. of } \\
\text { proteins }\end{array}$ & $\begin{array}{l}\text { No. of } \\
\text { peptides }\end{array}$ & $\begin{array}{l}\text { Avg. inf. } \\
\text { content }\end{array}$ & $\begin{array}{l}\text { Consensus } \\
\text { secondary } \\
\text { structure }\end{array}$ & Regular expression ${ }^{\mathrm{c}}$ & $\begin{array}{l}\text { Representative } \\
\text { PDBId-chainId- } \\
\text { ResPos }\end{array}$ \\
\hline \multicolumn{7}{|c|}{ A. Regular alpha-helices } \\
\hline S.4.1.0.11.0 & 3983 & 166,662 & 0.28 & НННННННН & $x(8)$ & 1jmr-a-4 \\
\hline S.4.1.0.11.2 & 2619 & 7773 & 0.30 & НННННННН & $x(3)-\{P\}(4)-x$ & 1 jmr-a-85 \\
\hline S.4.1.0.10.3 & 2647 & 6690 & 0.27 & ННHННHНH & $x(4)-\{P\}(3)-x$ & 1 jmr-a-86 \\
\hline S.4.1.0.12.6 & 1674 & 3454 & 0.29 & НННННННН & $x(8)$ & 1qj2-c-227 \\
\hline S.4.1.0.11.8 & 1458 & 2261 & 0.26 & НHНHНHНH & $x(4)-\{P\}(3)-x$ & 1gdg-a-154 \\
\hline \multicolumn{7}{|l|}{ B. Kinked-helices } \\
\hline S.4.1.0.11.5 & 2475 & 5711 & 0.26 & НННННННН & $x(7)-\{P\}$ & 1qj2-b-241 \\
\hline S.4.1.0.13.7 & 1502 & 2353 & 0.38 & НHНННННН & $x(6)-\{P\}-x$ & 1jmr-a-80 \\
\hline S.4.1.0.11.13 & 1179 & 1556 & 0.38 & НННННННН & $x(3)-\{P\}-x(2)-\{P\}-x$ & $1 q j 2-a-103$ \\
\hline S.4.1.0.9.20 & 1016 & 1287 & 0.40 & HHHHHHHH & $x(4)-\{P\}-x(2)-\{P\}$ & $1 \mathrm{qq} 8-\mathrm{a}-18$ \\
\hline \multicolumn{7}{|c|}{ C. Helix with a loop in the N-terminal region } \\
\hline S.12.1.1.11.1 & 3133 & 8257 & 0.52 & LHHНHНHН & $x(6)-\{P\}-x$ & 1jmr-a-21 \\
\hline S.12.1.0.13.11 & 1360 & 1966 & 0.43 & LHHHHHHH & $x(5)-\{P\}(2)-x$ & $1 q j 2-c-83$ \\
\hline S.12.1.0.11.15 & 1136 & 1491 & 0.42 & LHНHНHНH & $x(5)-\{P\}(2)-x$ & 1qj2-b-124 \\
\hline \multicolumn{7}{|c|}{ D. Helix followed by a loop in the C-terminal region } \\
\hline S.22.6.1.11.4 & 2641 & 6331 & 0.71 & HHHHHHLL & $x(3)-\{P\}(2)-x-\{P\}(2)$ & 1jmr-a-176 \\
\hline S.4.5.0.10.17 & 1021 & 1378 & 0.28 & HHHHHHHL & $x(3)-\{P\}(4)-x$ & 1qj2-a-25 \\
\hline S.4.5.0.13.21 & 937 & 1171 & 0.35 & HHHHHHLL & $x(4)-\{P\}(2)-x(2)$ & 1qj2-b-193 \\
\hline \multicolumn{7}{|l|}{ E. Beta strands } \\
\hline S.13.1.0.23.104 & 199 & 218 & 0.47 & ВВВВВBВB & $\mathrm{x}(6)-\{\mathrm{W}\}-\{\mathrm{M}\}$ & 1ef1-a-253 \\
\hline S.2.10.1.23.389 & 75 & 77 & 0.53 & BBBBBBBB & $\begin{array}{l}\{\text { PRW }\}-\{C W\}-\{\text { P }\}-\{C H P\}- \\
\{\text { FHMPW }\}-\{C S\}-\{C\}-\{C W\}\end{array}$ & $1 \mathrm{f} 1 \mathrm{~s}-\mathrm{a}-965$ \\
\hline S.20.7.1.22.470 & 66 & 66 & 0.52 & ВВВВВВВВ & $\begin{array}{l}\{\mathrm{M}\}-\{\mathrm{CPW}\}-\{\mathrm{PRW}\}-\{\mathrm{CDEHNP}\}- \\
\{\mathrm{Y}\}-\{\mathrm{PRW}\}-\{\mathrm{W}\}-\{\mathrm{N}\}\end{array}$ & $1 g w 5-s-3$ \\
\hline S.13.1.1.22.572 & 56 & 58 & 0.54 & ВВВВВВВВ & $\begin{array}{l}\{\mathrm{HW}\}-\{\mathrm{CP}\}-\{\mathrm{HQY}\}-\{\mathrm{ERW}\}-\{\mathrm{KR}\}- \\
\{\mathrm{ACEGH}\}-\{\mathrm{C}\}-\{\mathrm{MQ}\}\end{array}$ & 1cpt-a-335 \\
\hline S.13.5.1.24.705 & 47 & 50 & 0.56 & ВВВВВВВВ & $\begin{array}{c}\{\mathrm{CH}\}-\{\mathrm{CNR}\}-\{\mathrm{CDGP}\}-\{\mathrm{CDPQW}\}- \\
\{\mathrm{CKNP}\}-\{\mathrm{GQ}\}-\{\mathrm{CHKMNP}\}-\{\mathrm{CW}\}\end{array}$ & 1g72-a-520 \\
\hline
\end{tabular}

Summary and cluster contents for all detected structural clusters are available in the Supplementary Material, Tables S2 and S4, respectively.

a Cluster ID: for nomenclature of cluster ID, refer to Figure 5A, inset.

b Infomation content calculated as described. ${ }^{27}$

c Regular expression is given for each cluster as per PROSITE format. ${ }^{18}$ 
Table 3. Known Loop classes: summary of representative "structural" clusters corresponding to known classes of loops

\begin{tabular}{|c|c|c|c|c|c|c|c|c|c|}
\hline ClusterID $^{a}$ & $\begin{array}{l}\text { No. of } \\
\text { proteins }\end{array}$ & $\begin{array}{l}\text { No. of } \\
\text { peptides }\end{array}$ & $\begin{array}{l}\text { Avg. inf. } \\
\text { content }\end{array}$ & $\begin{array}{l}\text { Consensus sec- } \\
\text { ondary structure }\end{array}$ & Regular expression ${ }^{a}$ & $\begin{array}{l}\text { Representative } \\
\text { PDBId-chainId- } \\
\text { ResPos }\end{array}$ & $\begin{array}{l}\text { Standard LOOP } \\
\text { nomenclature }^{\mathrm{b}}\end{array}$ & $\begin{array}{l}\text { LOOP } \\
\text { class }^{c}\end{array}$ & $\begin{array}{l}\text { LOOP class } \\
\text { number }^{\mathrm{c}}\end{array}$ \\
\hline \multicolumn{10}{|c|}{ A. Known beta hairpins } \\
\hline S.1.6.1.6.19 & 995 & 1296 & 0.93 & BBLLLLLB & $x(2)-\{P\}-x-\{P\}-\{P V\}-\{P\}-x$ & 1jmr-a-51 & Type I[1-4] + G1 b-bulge 3:5 & $\begin{array}{l}\mathrm{bb}\{\mathrm{aag}\} \mathrm{bb} / \\
\mathrm{bb}\{\mathrm{aal}\} \mathrm{bb}\end{array}$ & $\begin{array}{l}\text { 3.1.1/3.1.2/ } \\
3.3 .1\end{array}$ \\
\hline S.23.6.1.4.32 & 507 & 618 & 0.91 & BBLLLLBB & $\{G\}-\{P\}-x-\{C\}-\{P W\}-x-\{P\}-x$ & 1qj2-b-668 & Type I[1-4] 4:4 & $\mathrm{bb}\{$ aaal $\} \mathrm{bb}$ & $\begin{array}{l}4.1 .1 / 4.1 .2 / \\
4.1 .3\end{array}$ \\
\hline S.27.9.1.5.79 & 261 & 287 & 1.10 & BBBLLBBB & $\begin{array}{l}x(2)-\{G P\}-\{C I M P T V W\}- \\
\{C I L P W Y\}-\{G P W\}-x-\{P W\}\end{array}$ & 1qj2-a-8 & Type I' 2:2 & $\mathrm{bb}\{\lg \} \mathrm{bb}$ & 2.1 .3 \\
\hline \multicolumn{10}{|c|}{ B. Known beta link } \\
\hline S.15.1.2.11.187 & 130 & 131 & 1.81 & LLLLLLLL & $\begin{array}{c}\{\text { P }\}-\{\text { EGHKMNY\}-\{CITW\}- } \\
\{\text { FGHMW\}-[ADEGHLNRS]- } \\
\{\text { CFHPR }\}-\{C G H R Y\}-\{Q W\}\end{array}$ & $1 e 79-a-46$ & Type II $+\mathrm{p}$ & $\mathrm{bb}\{\mathrm{pgp}\} \mathrm{bb}$ & 3.1 .4 \\
\hline \multicolumn{10}{|c|}{ C. Known beta-alpha } \\
\hline S.21.2.2.13.1083 & 36 & 38 & 2.46 & BBBLLHHH & $\begin{array}{l}\text { [AGILTV]-[ILV]-[AFIKLQV]- } \\
\text { [DG]-[AFGILSTVY]-[GS]- } \\
\text { \{DEKMQTW\}-[AFGIKLMSTV] }\end{array}$ & $1 \mathrm{kf6} 6-\mathrm{a}-8$ & $\mathrm{NAD}(\mathrm{P})$ binding loop & $\mathrm{bb}\{\mathrm{eab}\} \mathrm{aa}$ & 3.1 .1 \\
\hline \multicolumn{10}{|c|}{ D. Known alpha-alpha } \\
\hline S.17.3.1.10.147 & 148 & 160 & 0.80 & HHHLLHHH & $\begin{array}{l}\{C\}-\{C F M\}-x-\{C G P\}-\{C F I V\}-x- \\
\{C M P\}-\{P W Y\}\end{array}$ & 1ith-a-47 & $\mathrm{aBBa}$ & aa $\{b p\} a a$ & 2.1 .1 \\
\hline S.10.5.1.10.611 & 55 & 56 & 1.08 & HHHLLHHH & $\begin{array}{l}\text { \{CFHMPW\}-[ADEFIKLNQRTY]- } \\
\text { \{CEGIPV\}-[ADEGKNQRSY]- } \\
\{\text { DP\}-\{CHMP\}-\{CIRTVW\}- } \\
\{\text { FGMPW }\}\end{array}$ & 2 sqc-a-273 & $\mathrm{aGa}$ & aa\{1\}aa & 1.2 .3 \\
\hline \multicolumn{10}{|c|}{ E. Clusters that have been assigned different loop class numbers as well as different secondary structural flanks in the loop database } \\
\hline S.11.2.1.11.51 & 342 & 363 & 0.91 & HHLLLBBB & $x(2)-\{P\}-\{I M P T V W Y\}-\{P\}-x-\{H\}-x$ & 1qj2-b-718 & $\begin{array}{l}\text { Type I[1-4] + G1 b-bulge } 3: 5 \text { / } \\
\text { a(m)gaLb conformation }\end{array}$ & $\begin{array}{l}\mathrm{bb}\{\mathrm{aag}\} \mathrm{bb} / \\
\mathrm{a}\{\mathrm{g}\} \mathrm{bb}\end{array}$ & $\begin{array}{l}\text { 3.1.1/1.1.4/ } \\
1.1 .5\end{array}$ \\
\hline S.24.6.1.9.225 & 108 & 114 & 0.69 & HHLLHHHH & $\begin{array}{c}\{\mathrm{C}\}-\mathrm{x}-\{\mathrm{FIP}\}-\{\mathrm{MPVY}\}-\{\mathrm{CHMW}\}- \\
\{\mathrm{CH}\}-\{\mathrm{DGPSW}\}-\{\mathrm{CDEP}\}\end{array}$ & $1 \operatorname{cg} 5-\mathrm{a}-50$ & $\mathrm{aBa} / \mathrm{New}$ & $\begin{array}{l}\text { aaaa/ } \\
\text { bb\{aab\}aa }\end{array}$ & 1.1.3/3.3.1 \\
\hline \multicolumn{10}{|c|}{ F. Clusters that have been assioned different loop class numbers but similar secondary structural flanks in the loop databasec } \\
\hline S.9.1.1.5.48 & 342 & 372 & 0.95 & HHHHLLLL & $x(3)-\{P\}-\{C F L M P V Y\}-\{P\}-x(2)$ & 1qj2-b-407 & $\mathrm{a}(\mathrm{m}) \mathrm{gaLb}$ conformation & $\begin{array}{l}\text { aa }\{g\} b b / \\
\text { aa\{gp\}bb }\end{array}$ & $\begin{array}{l}1.1 .4 / 2.2 .2 / \\
1.1 .2\end{array}$ \\
\hline S.14.1.1.11.363 & 80 & 81 & 0.84 & ВВВНHНHН & $\begin{array}{l}\{\text { HY }\}-\{C H W\}-\{\text { DGNW }\}-[C D G- \\
\text { HKNPSTV]-\{CH }\}-\{C H M W Y- \\
\{\text { IPR }\}-\{C D E P T\}\end{array}$ & 1gte-a-302 & $\mathrm{ba}(\mathrm{n})$ conformation & $\begin{array}{l}\text { bb\{\}aa/ } \\
\text { bb\{p\}aa }\end{array}$ & $0.1 .4 / 1.1 .2$ \\
\hline
\end{tabular}

a Refer to footnotes to Table 2.

The standard loop nomenclature is provided if matches are found for one or more peptides from a given cluster with the known loop categories. ${ }^{1,7}$

c The LOOP class nomenclature is provided if matches are found for one or more peptides from a given cluster with the LOOP database. ${ }^{9}$ 
Table 4. Examples of novel structural motifs in loop regions

\begin{tabular}{|c|c|c|c|c|c|c|}
\hline ClusterID $^{a}$ & $\begin{array}{l}\text { No. of } \\
\text { proteins }\end{array}$ & $\begin{array}{l}\text { No. of } \\
\text { peptides }\end{array}$ & $\begin{array}{l}\text { Avg. inf. } \\
\text { content }^{a}\end{array}$ & $\begin{array}{l}\text { Consensus } \\
\text { secondary } \\
\text { structure }\end{array}$ & Regular expression ${ }^{\mathrm{a}}$ & $\begin{array}{l}\text { Representative } \\
\text { PDBId-chainId- } \\
\text { ResPos }\end{array}$ \\
\hline $\begin{array}{l}\text { A. New beta hai } \\
\text { S.15.6.1.5.574 }\end{array}$ & pin 55 & 58 & 0.84 & BBLLLBBB & $\begin{array}{l}\{\text { HLP\}-\{CGM\}-\{CHIPVWY\}- } \\
\text { [ADEGHKNPRSTV]-[DEGHK- } \\
\text { MNQRST]-\{CGLP\}- } \\
\text { \{DGHKMP\}-\{FMPW\} }\end{array}$ & $1 e 3 m-a-493$ \\
\hline $\begin{array}{l}\text { B. New beta-al, } \\
\text { S.3.1.1.15.559 }\end{array}$ & 58 & 59 & 0.76 & BBBLLHHH & $\begin{array}{l}\{\mathrm{CHMW}\}-\{\mathrm{DEG}\}-\{\mathrm{CGM}\}- \\
\text { \{DHKQTW\}-[ADEGHN- } \\
\text { PQRST]-\{CFNW\}-\{HMV\}-\{MPR }\}\end{array}$ & 5eau-a-386 \\
\hline S.13.1.1.21.997 & 40 & 40 & 1.20 & BBBLLLHH & $\begin{array}{l}\text { \{ACMQ\}-[ACEFGIKLRTVW]- } \\
\text { [ADFHIKLPQRTV]-[ADEIKL- } \\
\text { PRTV]-[ACIKLMNQRTVY]- } \\
\text { [DEHLNPRST]-\{CFGHILW\}- } \\
\text { \{CFHILMY\} }\end{array}$ & $1 a 62-a-62$ \\
\hline \multicolumn{7}{|c|}{ C. New alpha-beta } \\
\hline S.11.2.1.12.397 & 75 & 76 & 1.08 & HHHLLBBB & $\begin{array}{l}\{\mathrm{CMPWY}\}-\{\mathrm{HMVWY}\}-\{\mathrm{P}\}-[\mathrm{EGK}- \\
\text { MNQRSY]-\{HPQW\}-\{CDNW\}- } \\
\{\mathrm{CDMNW}\}-\{\mathrm{EQ}\}\end{array}$ & $1 j 8 w-b-326$ \\
\hline S.11.7.2.8.529 & 56 & 62 & 1.62 & HHHLLLBB & $\begin{array}{l}\text { [AEFIKLMQRTVY]-\{CIPTV\}- } \\
\text { \{PQW\}-[DGKN]-[ACEFGIK- } \\
\text { MRVY]-\{ACFHMWY\}-\{NPQW\}- } \\
\text { [AFGILPQVY] }\end{array}$ & $112 r-a-357$ \\
\hline S.15.3.1.7.940 & 42 & 42 & 1.08 & HHHLLLBB & $\begin{array}{l}\{\text { ACPRW }\}-\{C F G N T W\}-\{C F G- \\
\text { HPWY\}-[DFGKLNRY]-\{CGMP\}- } \\
\text { [AEFIKLNQSTVY]-\{CFW\}- } \\
\{\text { CDHT }\}\end{array}$ & $1 j m r-a-45$ \\
\hline \multicolumn{7}{|c|}{ D. New alpha-alpha } \\
\hline S.3.4.1.13.210 & 119 & 120 & 1.05 & HHLLLHHH & $\begin{array}{l}\{\mathrm{CW}\}-\{\mathrm{PW}\}-[\mathrm{ACDEGHKNQRS}]- \\
\{\mathrm{DEGHP}\}-\{\mathrm{CIVW}\}\}-\{\mathrm{H}\}- \\
\{\mathrm{CMW}\}-\{\mathrm{CGPW}\}\end{array}$ & 1qj2-b-598 \\
\hline S.17.6.1.10.264 & 101 & 102 & 0.73 & HHHLLHHH & $\begin{array}{l}x-\{C V W\}-\{C I P W\}-\{N P\}- \\
\{C I M Q R V W\}-\{C M N W\}- \\
\{C P V W Y\}-\{C P\}\end{array}$ & 1a59-a-88 \\
\hline \multicolumn{4}{|c|}{ E. New long loops } & LLLLLLLL & $\{\mathrm{DH}\}-[\mathrm{ACDGKLNQRSTW]- \{ C}\}-$ & 1qla-c-13 \\
\hline S.1.6.1.6.297 & 93 & 94 & 1.09 & LLLLLLLL & $\begin{array}{c}\{\mathrm{CGP}\}-\{\mathrm{CEIPTV}\}-\{\mathrm{CP}\}-\{\mathrm{DH}\}-\mathrm{x} \\
\{\mathrm{N}\}-\{\mathrm{DG}\}-\{\mathrm{FIMPQ}\}-\{\mathrm{CHN}\}-\{\mathrm{PY}\}- \\
{[\mathrm{DGHN}]-\{\mathrm{FGP}\}-\{\mathrm{CN}\}}\end{array}$ & 1pgj-a-243 \\
\hline S.3.2.1.16.294 & 89 & 94 & 0.65 & LLLLLLLL & $\begin{array}{c}x-\{C D\}-\{G Y\}-\{C E G M N S\}-[A D E- \\
\text { HKMNPRSTV]-\{C\}-\{PW\}-\{HP\} }\end{array}$ & 1qbk-b-327 \\
\hline
\end{tabular}

These "structural" clusters are neither superimposable nor have any overlap with the reported categories of Loops. ${ }^{17,9}$ Summary of all detected functional clusters available in Supplementary Material Table S3.

a See footnotes to Table 2 .

content (Table 5). For structural clusters, we analyzed the dependence of the information content on cluster size. Smaller clusters showed higher information content than the larger ones (Figure 4). The lowest information content that is possible for a cluster is shown by a bold line. Interestingly, all the clusters show information content greater than the lowest possible, while some clusters show very high information content for their size. This may be a result of requirement of certain amino acid residue or residue types to attain a certain conformation at that position in the peptide.

\section{Structural clusters}

A total of 10,696 clusters were declared as structural clusters with their sizes ranging from five to 166,662 peptides per cluster. Examination of secondary structure assignment of the peptides of a cluster reveals that the majority of the peptides of a cluster had similar assignments. Each cluster was made up of either regular secondary structures such as helix or sheet, or non-regular structures of loops with or without the secondary structures. This has resulted in several clusters of helices, of beta sheets and of loops. It is expected that a cluster of helices would be more similar to another cluster of helices than that of beta strands. Likewise, it is of interest to understand the level of geometric similarity between the clusters. To this end, the structural clusters were further grouped based on the closeness of their centroids 
in the 56-dimensional representation using the geometric invariants. Thus, the clusters were hierarchically clustered into groups and supergroups. Clusters that belong to a group are highly similar in geometry while clusters of a supergroup are broadly similar. This provides a clear-cut relationship between the different clusters, which has now been implied in the nomenclature of the structural clusters (see inset of Figure 5(a)). A representative dendrogram is shown (Figure 5(a)) to indicate the hierarchical relationship between the structural clusters belonging to supergroup number 25, the supergroup with the smallest number of clusters. A heat map is shown with one representative cluster from each supergroup (Figure 5(b)).

\section{Helices}

Several distinct clusters were detected which were made up of helices such that all eight amino acids in the octapeptides are assigned as helix in the respective PDB structure files. ${ }^{28,29}$ The largest cluster S.4.1.0.11.0 was of 166,662 peptides drawn from 3983 peptide chains and contains several overlapping peptides (Table 2). These appear to be regular helices where adjacent peptides, starting at $i, i+1, i+2$, etc., are superimposable. The average dihederal angles of this cluster follow values close to those for an ideal helix and do not vary with the residue position within the peptide. Except for the cluster S.4.1.0.11.0, all other helix clusters (i) do not contain adjacent peptides and (ii) show some deviation in the average phi-psi angles from those for an ideal helix. This deviation may be near the $\mathrm{C}$ terminus, $\mathrm{N}$ terminus or center of the peptide. For example, the clusters S.4.1.0.11.2, S.4.1.0.10.3, S.4.1.0.12.6 show small but noticeable deviation, yet they have been assigned helix as secondary structure in the PDB files. ${ }^{28,29}$ On the other hand, the clusters S.4.1.0.11.5, S.4.1.0.13.7, S.4.1.0.11.13, S.4.1.0.9.20 show significant deviation in the middle region, which amounts to a break in helix continuity. These peptides have been termed as kinked helices or helix-loop-helix with zero residues in the loop. For example, the cluster S.4.1.0.11.13 shows a break in the helicity at the third amino acid residue (Figure 6A). The majority of the clusters of regular helices as well as kinked helices belong to group 4.1. In fact, the group 4.1 contains 307 clusters, which together account for 214,971 peptides. In addition, we detected several large clusters of helices with one to two residue loop flanks at the $\mathrm{N}$ terminus or $\mathrm{C}$ terminus of the octapetide (Table 2). For example, the cluster S.12.1.1.11.1 is made up of octapeptides with a one residue loop followed by a seven residue helix. This indicates the structural conservation of the loop residue in addition to the helix (Figure 6B). The clusters of helices with loop residues in the $\mathrm{N}$ terminus belong to group 9.2 while those with loop residues in the $\mathrm{C}$ terminus belong to groups 21.1 and 21.3. These clusters seem to have arisen as a result of a preferred way of terminating the helix at the $\mathrm{C}$ or N-terminal end of the helix. Interestingly, there is noticeable variation in the sequence conservation pattern of these clusters. For example, the cluster of regular helices, S.4.1.0.11.2, does not show any sequence conservation pattern with any amino acid being acceptable at any of the positions within the peptide. All other clusters of helices, kinked helices as well as helices with $\mathrm{N}$ or $\mathrm{C}$-terminal loops exclude proline at one to four positions where the locations of proline exclusion vary from one cluster to another (Table 2).

\section{Beta strands}

We detect more than 200 clusters made up of beta strands with all eight amino acids being assigned as beta strand in the respective PDB structure files. These clusters rarely contain adjacent peptides and are significantly smaller compared to the clusters of helices, with the largest cluster of beta strands being made up of 218 peptides (Table 2). Moreover, these clusters belong to different supergroups, indicating the diversity in the geometry of the beta strands (Figure 5B). The sequence conservation pattern indicates that sheets exclude tryptophan as well as cysteine residues at several locations. This is in agreement with the known sequence preference pattern of strands. The plot of average torsion angles for the clusters indicates that the peptides in cluster S.13.1.0.23.104 are closer to an ideal beta strand, while the other clusters are made up of distorted or bent strands (for example, see Figure 6C). The secondary structure assignment of beta strand to these geometrically diverse clusters is based on fulfilment of a loosely defined criterion of dihederal angles or hydrogen bonds between adjacent strands.

\section{Loops}

A large number of clusters contained loop regions within the octapeptide. The number of residues assigned as loop residues in the PDB structure file varies from octapeptide to octapeptide even within the same cluster. This may be a result of ambiguity in assignment of secondary structure. $^{8}$ The largest cluster of loops, S.1.6.1.6.19, contains 1296 peptides from 995 proteins and contains up to five loop residues with the flanking beta strands (Figure 6D). The classical nomenclature for the peptides in this cluster is beta hairpin of type I [1-4]+ G1 b-bulge 3:5 ${ }^{1}$, which reflects that the number of residues in the loop is three or five, depending on the method of secondary structure assignment (Table 3). The second largest cluster of loops, S.23.6.1.4.32, was made up of 618 peptides from 507 proteins. These loops have been termed as beta hairpin type[1-4] $4: 4^{1}$. The geometry of these beta hairpin octapeptides seems to be more conserved than any of the beta strand 
Table 5. Summary of some representative "functional" clusters

\begin{tabular}{|c|c|c|c|c|c|c|c|}
\hline ClusterID $^{\mathbf{a}}$ & $\begin{array}{l}\text { No. of } \\
\text { proteins }\end{array}$ & $\begin{array}{l}\text { No. of } \\
\text { peptides }\end{array}$ & $\begin{array}{l}\text { Avg. } \\
\text { inf. } \\
\text { content }^{\mathbf{b}}\end{array}$ & Regular expression ${ }^{\mathbf{b}}$ & $\begin{array}{l}\text { Consensus } \\
\text { secondary } \\
\text { structure }\end{array}$ & $\begin{array}{l}\text { Most } \\
\text { likely } \\
\text { start pos. }\end{array}$ & $\begin{array}{l}\text { Representative } \\
\text { pdbid-chainid- } \\
\text { respos }\end{array}$ \\
\hline \multicolumn{8}{|c|}{ A. (Phosphotyrosine protein) phosphatases II: active site } \\
\hline F.c.45.1.4.6.6407 & 9 & 10 & 3.80 & $\begin{array}{l}\text { [CS]-[RS]-A-G-[AIV]-G-R- } \\
\text { [ST] }\end{array}$ & LLLLLHHH & 1522 & 1lar-a-1522 \\
\hline \multicolumn{8}{|c|}{ B. $2 \mathrm{Fe}-2 \mathrm{~S}$ ferredoxin-like: ligand binding site } \\
\hline F.d.15.4.4.15.7873 & 8 & 8 & 3.75 & $\begin{array}{l}\text { [LW]-P-[FY]-S-C-[HR]- } \\
\text { [AS]-G }\end{array}$ & LLLLLLLL & 37 & 1czp-a-37 \\
\hline F.d.15.4.4.12.12883 & 5 & 5 & 3.85 & $\begin{array}{l}\text { [AS]-G-[AS]-C-S-[ST]-C- } \\
\text { [ALV] }\end{array}$ & LLLLLLLL & 43 & $1 c z p-a-43$ \\
\hline \multicolumn{8}{|c|}{ C. $4 F e-4 S$ ferredoxins: ligand binding site } \\
\hline F.d.58.1.3.16.7872 & 7 & 8 & 2.95 & $\begin{array}{l}\text { [DEQ]-[AELS]-C-I- } \\
\text { [ADFGS]-[CD]-[AGM]- } \\
\text { [ALSV] }\end{array}$ & LLLLLLLH & 6 & 1dur-a-6 \\
\hline \multicolumn{8}{|c|}{ D. Acid proteases: active site } \\
\hline F.b.50.1.3.11.7870 & 8 & 8 & 3.35 & $\begin{array}{l}\text { [ILV]-D-[ST]-G-A-[DS]- } \\
\text { [DIY]-[IST] }\end{array}$ & BLLLLLLB & 24 & 1c6y-a-24 \\
\hline F.b.50.1.4.10.10386 & 5 & 6 & 3.60 & $\begin{array}{l}\text { [FIV]-D-T-G-[ST]-[NS]- } \\
\text { [DFN]-[FLT] }\end{array}$ & BBLLLLLB & 31 & $1 \mathrm{fkn}-\mathrm{a}-31$ \\
\hline F.b.50.1.4.9.3460 & 17 & 17 & 3.91 & $\begin{array}{l}\text { [FV]-D-T-G-S-[AS]-[DNV]- } \\
\text { [FLV] }\end{array}$ & BBLLLLLB & 31 & $1 d p j-a-31$ \\
\hline F.b.50.1.4.10.12872 & 5 & 5 & 3.88 & $\begin{array}{l}\text { [ALV]-D-[ST]-G-T(2)-[LN]- } \\
\text { [IL] }\end{array}$ & BLLLLLLB & 212 & $1 \mathrm{fkn}-\mathrm{a}-227$ \\
\hline \multicolumn{8}{|c|}{ E. Cupredoxins: copper ligands } \\
\hline F.b.6.1.3.12.12780 & 5 & 5 & 3.28 & $\begin{array}{l}\text { [KST]-H-[PS]-G-[KNS]-L- } \\
\text { [AP]-K }\end{array}$ & BBLLLLLH & 34 & $1 \mathrm{cc} 3-\mathrm{a}-34$ \\
\hline F.b.6.1.3.13.12777 & 5 & 5 & 3.02 & $\begin{array}{l}\text { [EKS]-H-[KPV]-G-[HKN]- } \\
\text { [LM]-[AP]-K }\end{array}$ & BBLLLLHH & 34 & $1 a z c-a-34$ \\
\hline F.b.6.1.4.13.12790 & 5 & 5 & 3.96 & $\begin{array}{l}\text { [AP]-K-[NV]-[AV]-M-G-H- } \\
\mathrm{N}\end{array}$ & LLLLLLBB & 40 & $1 \mathrm{azc}-\mathrm{a}-40$ \\
\hline F.b.6.1.4.18.7851 & 8 & 8 & 3.61 & $\begin{array}{l}\text { Y-[DGKST]-[FY]-[HY]-C- } \\
\text { [AEST]-P-H }\end{array}$ & BBBBLLLL & 80 & 1 pcs-a- 80 \\
\hline F.b.6.1.3.6.10337 & 6 & 6 & 3.07 & $\begin{array}{l}\text { [FIV]-C-[QST]-[FIY]-P-G-H- } \\
\text { [ACFIY] }\end{array}$ & BLLLLLLL & 111 & 1cuo-a-111 \\
\hline F.b.6.1.4.6.8856 & 7 & 7 & 3.50 & $\begin{array}{l}\text { [FIV]-C-[NST]-[FV]-[GP]- } \\
\text { [GT]-H-[CFISW] }\end{array}$ & BBLLLLLL & 111 & 1azc-a-111 \\
\hline F.b.6.1.4.11.12792 & 5 & 5 & 4.03 & [FMW]-H-L-H-G-H-[AD]-F & BBBLLLLB & 397 & 1gyc-a-397 \\
\hline \multicolumn{8}{|c|}{ F. Cysteine proteinases: loops that house catalytic triad residues } \\
\hline F.d.3.1.4.10.3457 & 17 & 17 & 3.70 & $\begin{array}{l}\text { Q-[GK]-[EGQSY]-[CS]- } \\
\text { [EG]-[GRS]-[ACS]-W }\end{array}$ & LLLLLLHH & 19 & $1 \mathrm{f} 2 \mathrm{a}-\mathrm{a}-19$ \\
\hline F.d.3.1.3.18.4855 & 13 & 13 & 2.86 & $\begin{array}{l}\text { [ILRV]-[DNT]-H-[AGRV]- } \\
\text { [LV]-[ALST]-[ALV]-[AV] }\end{array}$ & LLBBBBBB & 157 & $1 \mathrm{f} 2 \mathrm{a}-\mathrm{a}-157$ \\
\hline F.d.3.1.3.9.2416 & 22 & 22 & 3.50 & $\begin{array}{l}\text { N-S-W-[DGNT]-[AEKPT]- } \\
\text { [ADEGNPQT]-[HSW]-G }\end{array}$ & LLLLLLLL & 179 & $1 \mathrm{f} 2 \mathrm{a}-\mathrm{a}-175$ \\
\hline \multicolumn{8}{|l|}{ G. Cytochrome c } \\
\hline F.a.3.1.3.14.5808 & 11 & 11 & 2.80 & $\begin{array}{l}\text { C-[AKLMQ]-[AQT]-C-H- } \\
\text { [MRT]-[AFIV]-[DEQ] }\end{array}$ & HLLLLLLL & 14 & 1wej-f-14 \\
\hline \multicolumn{8}{|c|}{ H. EF-hand: calcium binding loop } \\
\hline F.a.39.1.3.5.523 & 36 & 63 & 2.69 & $\begin{array}{c}\text { D-\{CDGHPWY\}-[DN]- } \\
\text { [AEGHKNQRST]- } \\
\text { [DGNST]-[EGKN]- } \\
\text { \{GIPSVW\}-[CILV] }\end{array}$ & LLLLLLLL & 51 & 1 pva-a-51 \\
\hline \multicolumn{8}{|c|}{$\begin{array}{l}\text { I. Immunoglobulin: hypervariable regions } \\
\text { L1 regions }\end{array}$} \\
\hline F.b.1.1.3.11.1485 & 30 & 30 & 2.90 & $\begin{array}{l}\text { [AET]-S-[EQ]-[DEGNS]- } \\
\text { [IV]-[DGINRSTY]- } \\
\text { [GIKNST]-[ADFGKNRSY] }\end{array}$ & BLLLLLLL & 25 & 1lk3-1-24 \\
\hline F.b.1.1.3.11.3448 & 17 & 17 & 3.33 & $\begin{array}{l}\text { A-[GS]-[EGQ]-[DGN]-[IT]- } \\
\text { [HKNSY]-[DKNST]- } \\
\text { [FNRY] }\end{array}$ & BLLLLLLL & 25 & 1wej-1-25 \\
\hline
\end{tabular}


Table 5 Continued

\begin{tabular}{|c|c|c|c|c|c|c|c|}
\hline ClusterID $^{a}$ & $\begin{array}{l}\text { No. of } \\
\text { proteins }\end{array}$ & $\begin{array}{l}\text { No. of } \\
\text { peptides }\end{array}$ & $\begin{array}{l}\text { Avg. } \\
\text { inf. } \\
\text { content }^{\mathbf{b}}\end{array}$ & Regular expression ${ }^{\mathbf{b}}$ & $\begin{array}{l}\text { Consensus } \\
\text { secondary } \\
\text { structure }\end{array}$ & $\begin{array}{l}\text { Most } \\
\text { likely } \\
\text { start pos. }\end{array}$ & $\begin{array}{l}\text { Representative } \\
\text { pdbid-chainid- } \\
\text { respos }\end{array}$ \\
\hline F.b.1.1.3.9.10239 & 6 & 6 & 3.39 & $\begin{array}{l}\text { [AF]-[ST](3)-[MV]-[NRSY]- } \\
\text { [SY]-[LMV] }\end{array}$ & BLLLLLLL & 25 & 1baf-1-25 \\
\hline F.b.1.1.4.11.10249 & 6 & 6 & 3.58 & $\begin{array}{l}\text { A-S-[KQ]-[DS]-I-[NRS]- } \\
\text { [HKNS]-[FSY] }\end{array}$ & BLLLLLLL & 25 & 1axs-a-25 \\
\hline F.b.1.1.4.17.7805 & 8 & 8 & 3.71 & $\begin{array}{l}\text { [APS]-[ST]-[KQ]-[AS]-[IL]- } \\
{[\mathrm{LV}]-[\mathrm{HN}]-[\mathrm{ES}]}\end{array}$ & BLLLLLLL & 25 & 1gpo-1-25 \\
\hline \multicolumn{8}{|l|}{ J. L2 regions } \\
\hline F.b.1.1.2.16.262 & 103 & 103 & 2.45 & $\begin{array}{l}\{\text { CHMPV\}-[ADIMSTV]- } \\
\text { [DKNST]-[HIKNQRSTY]- } \\
\text { [LQRS]-\{CKNRSTW\}- } \\
\text { [ADEIPSTY]-[EG] }\end{array}$ & LLLLLLLL & 50 & $11 k 3-1-49$ \\
\hline F.b.1.1.2.17.7798 & 8 & 8 & 2.36 & $\begin{array}{l}\text { [ADRY]-[ADNT]-[AST]- } \\
\text { [MNRS]-[LR]-[AHPR]- } \\
\text { [DS]-[EG] }\end{array}$ & LLLLLLLL & 50 & 1baf-1-49 \\
\hline F.b.1.1.3.10.12585 & 5 & 5 & 2.76 & $\begin{array}{l}\text { [KY]-[EGY]-[ADT]-[NS]- } \\
\text { [NRS]-[LR]-[AEH]-S }\end{array}$ & LLLLLLLL & 50 & 1fbi-1-49 \\
\hline F.b.1.1.3.15.4401 & 14 & 14 & 2.79 & $\begin{array}{l}\text { [ADGKLNWY]-[ATV]- } \\
\text { [KST]-[IKNST]-[LR]-[AEF- } \\
\text { GHRV]-[ELST]-[EGW] }\end{array}$ & LLLLLLLL & 50 & 1bql-1-49 \\
\hline \multicolumn{8}{|l|}{ K. L3 regions } \\
\hline F.b.1.1.2.9.530 & 62 & 62 & 2.49 & $\begin{array}{l}\text { [GHINRSTWY]-\{CEF- } \\
\text { MPRV\}-[ADEGHNRST]- } \\
\text { \{CEGMNQT\}-P-[FIL- } \\
\text { PRWY]-[AST]-F }\end{array}$ & LLLLLLLL & 91 & 11k3-1-90 \\
\hline F.b.1.1.3.9.6366 & 10 & 10 & 2.77 & $\begin{array}{l}\text { [DGHNSVY]-[DFKNSY]- } \\
\text { [DENRST]-[ADHLNVW]- } \\
\text { P-[FLPRY]-T-F }\end{array}$ & LLLLLLLL & 91 & 1dee-a-91 \\
\hline F.b.1.1.3.9.7022 & 9 & 9 & 3.02 & $\begin{array}{l}\text { [CDGS]-[GHNQWY]- } \\
\text { [AEST]-[DILNY]-P- } \\
\text { [FLPRWY]-T-F }\end{array}$ & LLLLLLLL & 91 & 1cic-a-91 \\
\hline \multicolumn{8}{|l|}{ L. H1 regions } \\
\hline F.b.1.1.3.11.12613 & 5 & 5 & 3.45 & $\begin{array}{l}\text { [AV]-[ST]-G-[FY]-[NS]-[IL]- } \\
{[\mathrm{KT}]-[\mathrm{DEST}]}\end{array}$ & BBLLLHHH & 24 & 1f58-h-24 \\
\hline F.b.1.1.4.13.6374 & 10 & 10 & 3.59 & $\begin{array}{l}\text { [AV]-S-G-[FI]-[LS]-[FL]- } \\
\text { [IRST]-[CDGNRST] }\end{array}$ & BBLLLLLL & 24 & $1 a 2 y-b-24$ \\
\hline F.b.1.1.3.13.453 & 69 & 69 & 3.29 & $\begin{array}{l}\text { [ST]-[GN]-[FLY]-[ADI- } \\
\text { MSTV]-F-[DIMNRST]- } \\
\text { [DEGKNRST]-[FHNSY] }\end{array}$ & BLLLHHHL & 25 & 1lk3-h-25 \\
\hline \multicolumn{8}{|l|}{ M. $\mathrm{H} 2$ regions } \\
\hline F.b.1.1.3.4.4042 & 15 & 15 & 2.62 & $\begin{array}{l}\text { [DLNVY]-[ADEGNSY]- } \\
\text { [INST]-[DG]-[DGNRSVY]- } \\
\text { [PST]-[EIKNSTVY]-[FY] }\end{array}$ & BLLLLBBB & 52 & $12 \mathrm{e} 8-\mathrm{h}-52$ \\
\hline \multicolumn{8}{|c|}{ N. Trypsin-like serine proteases: loops housing the catalytic triad residues } \\
\hline F.b.47.1.3.14.796 & 45 & 47 & 3.02 & $\begin{array}{c}\text { [ILV]-[ILMV]-[ST]-A-[AG]- } \\
\text { H-[CT]-[DIKLMVWY] }\end{array}$ & BBBLHHHH & 52 & 1fi8-a-52 \\
\hline F.b.47.1.3.14.10109 & 6 & 6 & 3.45 & $\begin{array}{l}\text { [FILV]-[ILV]-[ST]-A-[AHT]- } \\
\text { H-C-[FGLY] }\end{array}$ & ВВВННННН & 52 & 1 arb-a-52 \\
\hline F.b.47.1.3.11.4382 & 14 & 14 & 2.94 & $\begin{array}{l}\text { [DGKPQRS]-[DEFKNR]- } \\
\text { [NRT]-[FIKLY]-[DLNQS]- } \\
\text { [FNY]-D-[IV] }\end{array}$ & LLLLLLLL & 96 & 1fi8-a-96 \\
\hline F.b.47.1.3.11.8701 & 7 & 7 & 2.80 & $\begin{array}{l}\text { [AKPS]-[AGLQSY]- } \\
\text { [LNQST]-[EHINT]- } \\
\text { [ADILN]-[NQ]-D-[IL] }\end{array}$ & LLLLLLLL & 96 & 1aut-c-96 \\
\hline F.b.47.1.3.13.616 & 56 & 56 & 3.46 & $\begin{array}{l}\text { [CEFGL]-[AEFHKMN- } \\
\text { PQRS]-[DFG]-[DS]- } \\
\text { [AGS]-G-[GST]-[APS] }\end{array}$ & LLLLLLLB & 191 & $\begin{array}{l}\text { 1fi8-a-191, 1arb- } \\
\text { a-190, 1aut-c-191 }\end{array}$ \\
\hline
\end{tabular}

a Cluster ID indicates: F, functional cluster; SCOP superfamily code ${ }^{31}$ between the first and fourth dot, average information content of the cluster between the fourth and fifth dot, $\mathrm{C}^{\alpha 1}-\mathrm{C}^{\alpha 8}$ distance between the fifth and sixth dot and a unique identification number for each cluster after the sixth dot. See the legend to Table 2 for unique id.

b See footnotes to Table 2. The known functional amino acid residues are shown in bold font. 


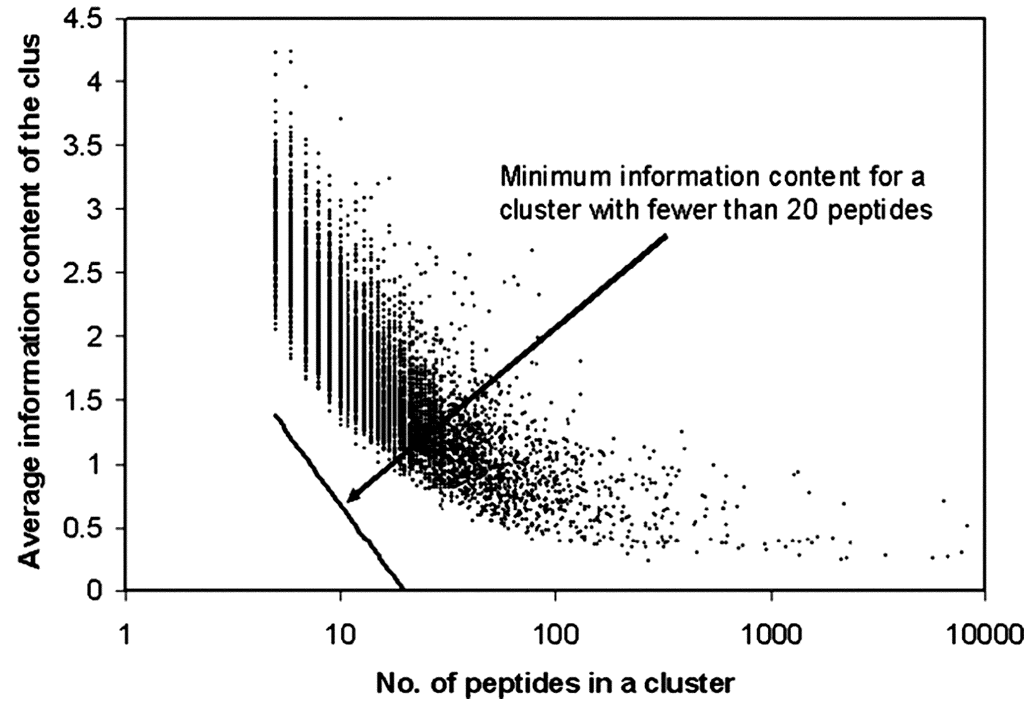

Figure 4. Average information content versus cluster size for structural clusters. octapeptides. We decided to compare our clusters with some of the broad-based loop classification databases ${ }^{8,9,32}$ in addition to the specialized classification of certain categories of loops. ${ }^{1,4}$ To that end, each of the peptides in our clusters was scanned against the Loop ${ }^{8}$ and Sloop ${ }^{32}$ database. A total of 341 of the clusters showed at least one match with the known classes of loops described in the Loop database, while 4077 of the clusters show at least one match with those of the SLoop database. For example, peptides from cluster S.1.6.1.6.19 have been assigned beta hairpin class no 3.1.1, while those from cluster S.23.6.1.4.32 are assigned beta hairpin class number 4.1.1 in the Loop database (Table 3). Examples of clusters with known Loop nomenclature are shown in Figure 6D-F. However, in several cases, two peptides from a cluster match with two distinct class numbers or even a totally different category of loops in the Loop database. For example, peptides 1NBA-A-220 and 3DFR-A39 belong to cluster S.14.1.1.11.363. These two peptides are assigned beta-alpha class numbers 0.1.4 and 1.1.2, respectively, in the Loop database. This indicates that the first peptide has a zero residue loop joining a beta strand to an alpha helix, while the second peptide has a one residue loop. We find the two peptides to belong to a common cluster and find them to be superimposable with an RMSD of 0.5 (Figure 6G). Likewise, peptides 1TGS-I-25 and 1FBA-A-178 of cluster S.11.2.1.11.51 are classified as beta-hairpins and alpha-beta, respectively, in the Loop database. We found these peptides to be superimposable with an RMSD of 0.5 (Figure $6 \mathrm{H}$ ). We conjecture that the discrepancy in class assignment, in the Loop database, has arisen due to differences in the secondary structure assignment to the two peptides, resulting in differences in loop categories or the number of residues assigned as the loop residues. A conventional loop classification algorithm then compares loops of zero residues separately and those of one residue separately. ${ }^{8,9}$ In other words, zero-residue loops are never com- pared with one-residue loops and thus different Loop classes are assigned to potentially superimposable Loop regions.

In addition to the known Loop classes, we detected several clusters for which Loop classes have not been assigned (Table 4$).{ }^{8}$ For example, clusters S.3.4.1.13.210, S.21.1.1.7.137, and S.3.1.1.15.559 appear to be new clusters of $\alpha$-turn- $\alpha$. $\beta$-hairpin and $\beta$-turn- $\alpha$, respectively. These newly discovered classes are shown in Figure 6I-L. These clusters are neither superimposable nor have any overlap in terms of common peptides with any of the reported clusters. ${ }^{7,9}$

A majority of the large structural clusters of loops do not show strong a sequence conservation pattern. However, some of the loop clusters, such as S.4.1.0.11.10, do show a preference for Gly or Asp residues at certain locations, while some loops, such as S.1.6.1.6.19 and S.23.6.1.4.32, show exclusion of certain residues such as Gly, Pro, Trp or Cys at certain locations (Table 4). These constraints may have arisen as certain types of conformations are not attainable by certain amino acids.

\section{Functional clusters}

A summary of representative functional clusters is given in Table 5. The SCOP superfamily name, information content and $d_{\alpha_{\alpha^{1}}-C_{\alpha^{8}}}$ have been incorporated in the cluster ID of the functional clusters. The functional clusters were typically made up of peptides in the loop regions, which take up a specific configuration due to the overall structural and environmental constraints of that superfamily. The sequence conservation pattern of 305 functional clusters out of 2207 match with the PROSITE sequence signature ${ }^{18,33}$ of the respective SCOP superfamily. The rest of the functional clusters' sequence motifs are not described in the PROSITE database. We describe the potential biological significance of some of the functional clusters below. 
(a) $\quad$ S.25.0.2.10.6117

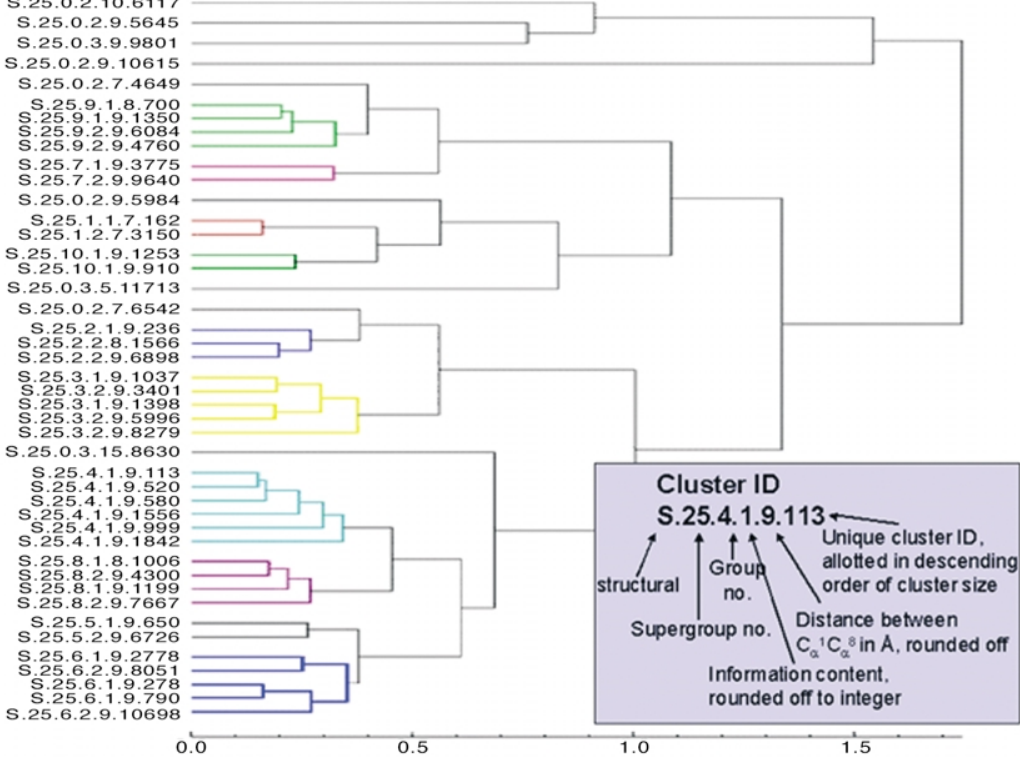

(b)
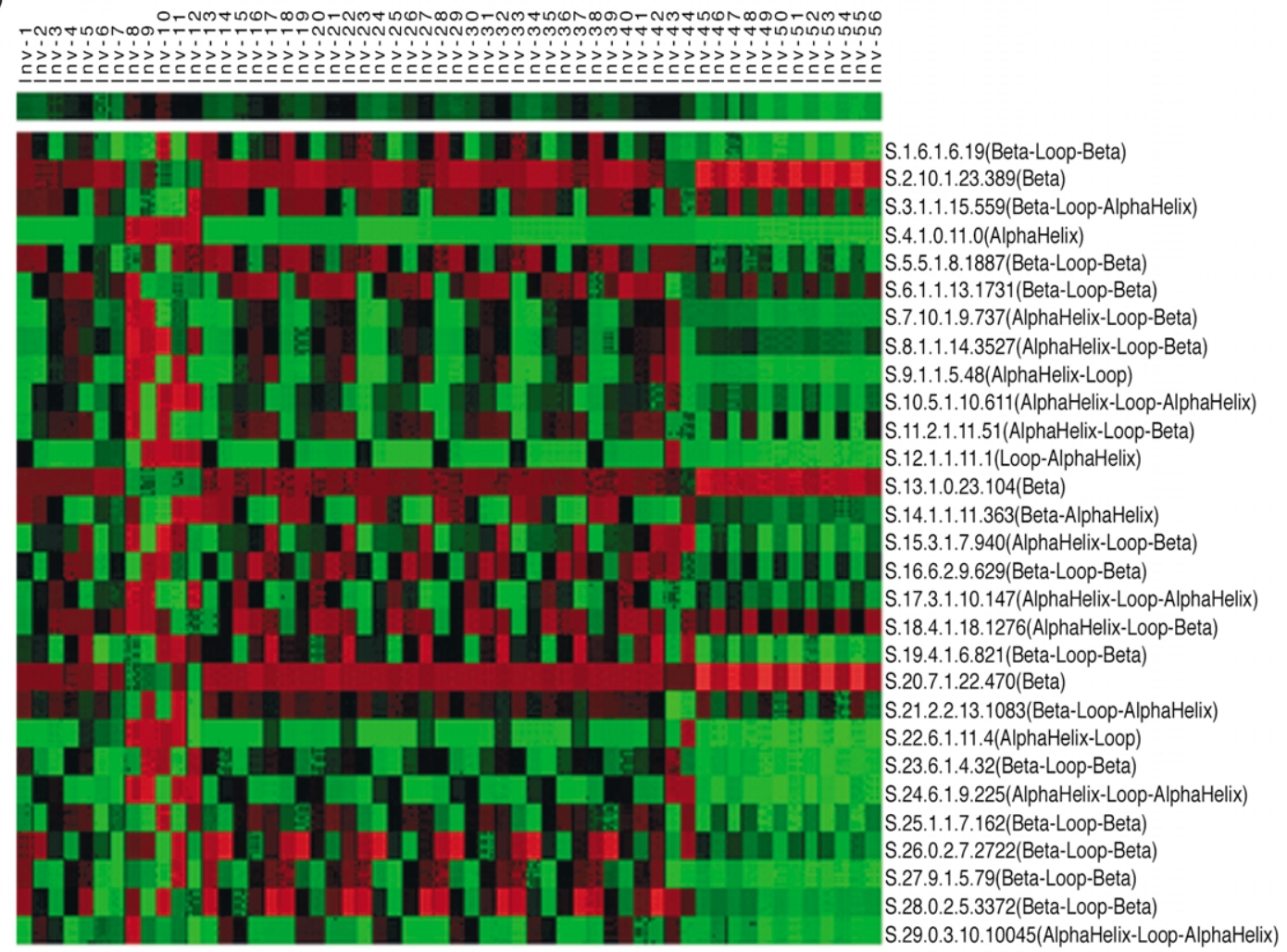

Figure 5. a, A representative dendrogram defining the hierarchy between structural clusters belonging to supergroup number 25 . The dendrogram is generated by using the 56 -dimensional centroid of each of the clusters. $b$, Heatmap showing the centroids of one representative cluster from each supergroup. The numerical values of the centroids are color-coded for each geometric invariant to show high (red), medium (grey) and low (green) values compared to the average value for a given geometric invariant. Consensus secondary structure of the chosen cluster is indicated in brief alongside the cluster ID.

\section{Aspartic proteases}

Aspartic proteases are characterized by an active site made up of two aspartate residues, drawn from a single peptide chain in non-retroviral proteins or drawn from two chains of the homodimer of the retroviral proteases. ${ }^{34}$ We detected four distinct clusters that house the catalytic aspartate residue as the second amino acid residue in the octapeptide. Three clusters, F.b.50.1.4.9.3460, 

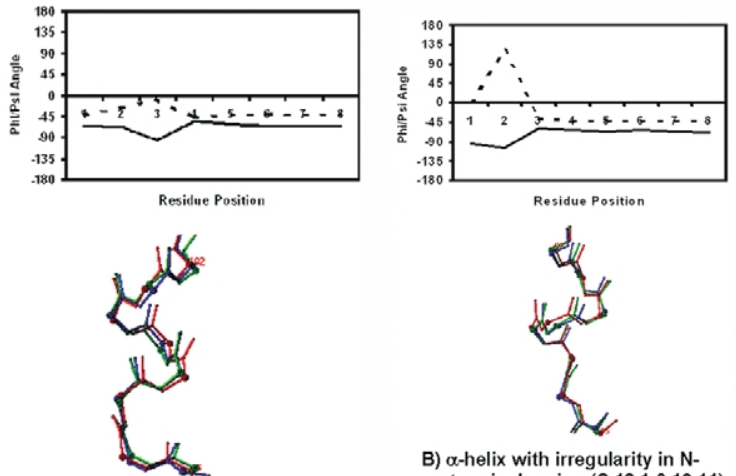

A) Kinked helix (S.4.1.0.11.13)
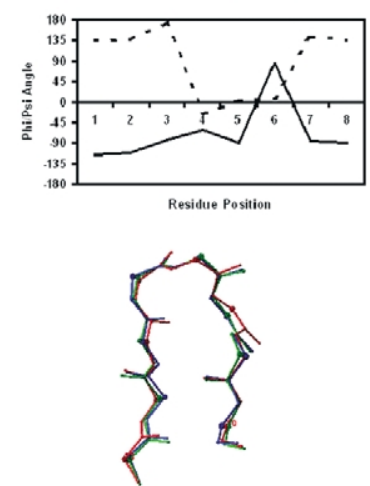

D) Known $\beta$-hairpin (S.1.6.1.6.19)
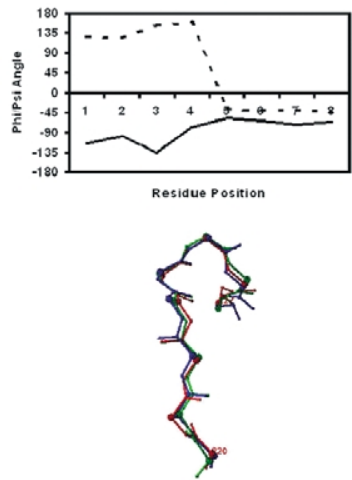

G) Ambiguity in Loop database (S.14.1.1.11.363)
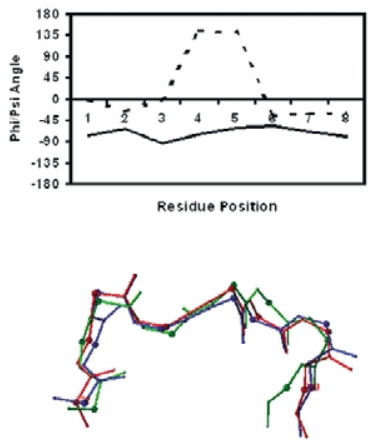

J) New $\alpha$-turn- $\alpha$ (S.17.6.1.10.264)

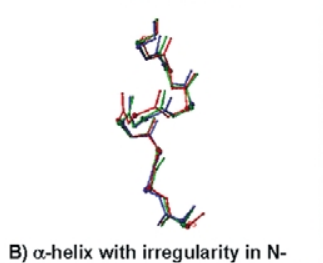

terminal region (S.12.1.0.13.11)
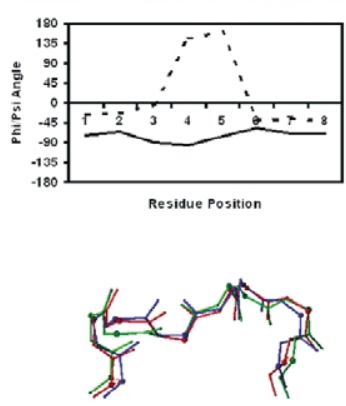

E) Known $\alpha-$ turn- $\alpha(S \cdot 17.3 \cdot 1 \cdot 10.147)$

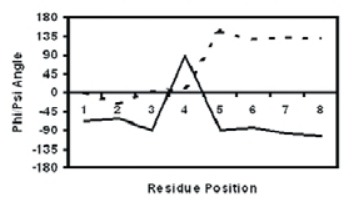

Resiaue position

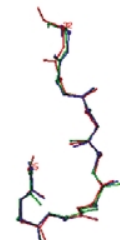

H) Ambiguity in Loop database (S.11.2.1.11.51)
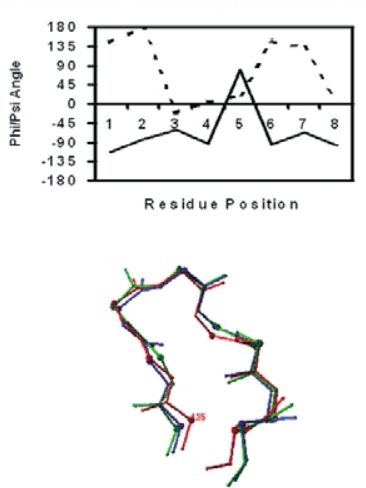

K) New $\beta$-hairpin (S.21.1.1.7.137)
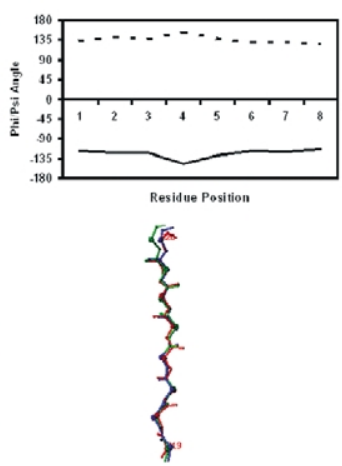

C) Twisted $\beta$-strand (S.2.10.1.23.389)
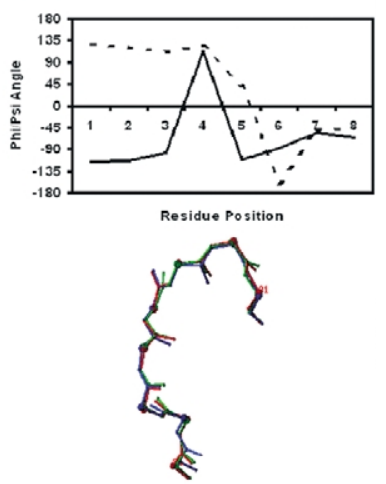

F) Known $\beta$-turn- $\alpha$ (S.21.2.2.13.1083)
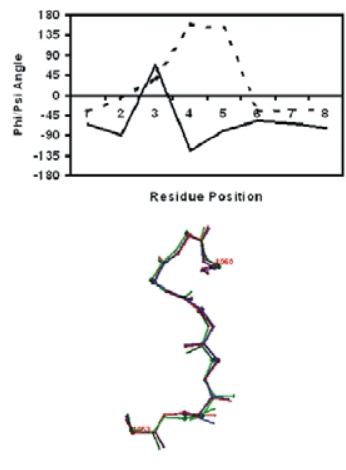

I) New $\alpha$-turn- $\alpha$ (S.3.4.1.13.210)
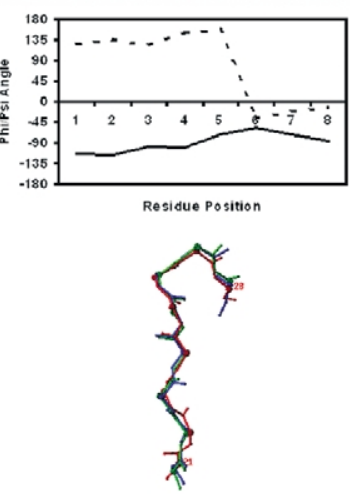

L) New $\beta$-turn- $\alpha$ (S.3.1.1.15.559)

Figure 6. Average phi-psi angle plots and superposed images for some representative structural clusters. Superposed images were generated using Swiss-PdbViewer. ${ }^{30}$ A, Kinked-helix (S.4.1.0.11.13) (1HLB-A-95,2GST-A120,1MTY-D-329,0.5,0.8); B, $\alpha$-helix with irregularity in N-terminal region (S.12.1.0.13.11) (3SDH-A-85,1GHS-A203,1HSL-A-141,0.5.0.7); C, twisted $\beta$-strand (S.2.10.1.23.389) (1DYP-A-219, 1DGW-X-281,1C8U-A-87,0.9,0.8); D, 

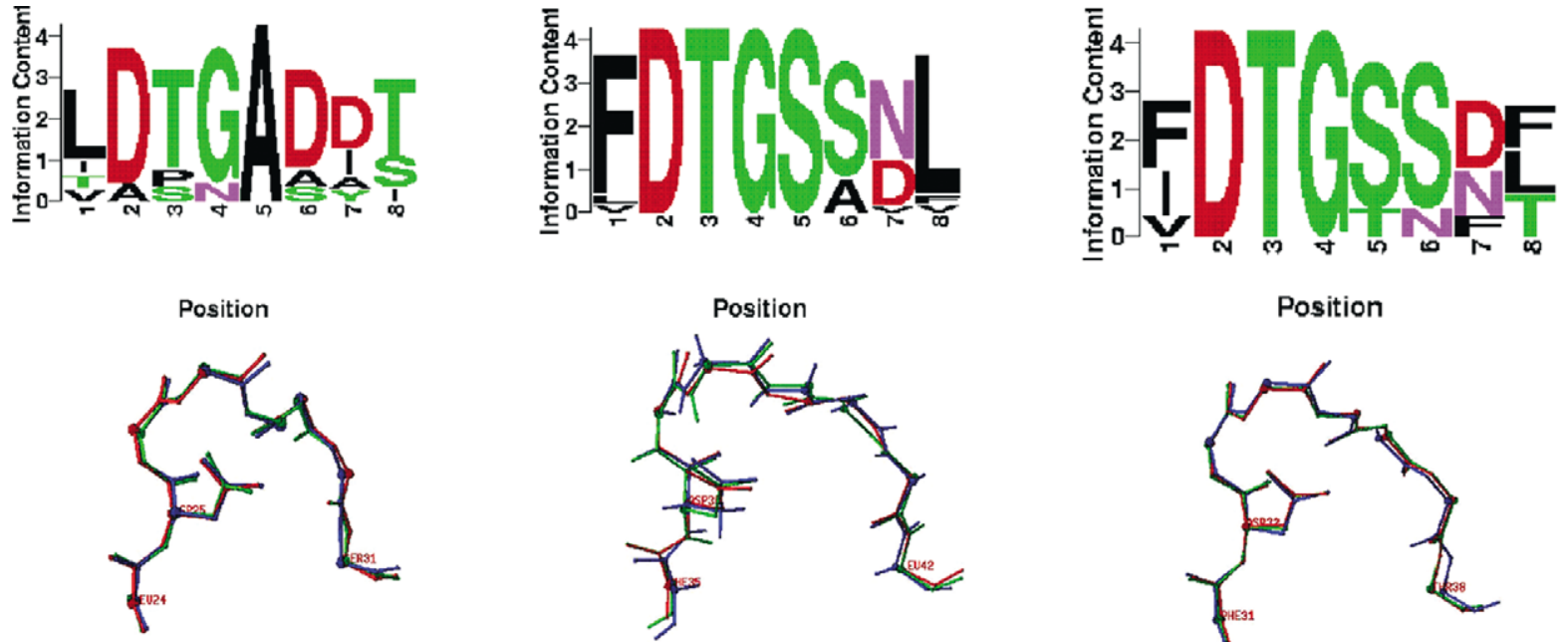

A. Acid Proteases: Active site loop conformation I (F.b.50.1.3.11.7870)

B. Acid Proteases: Active site loop conformation II (F.b.50.1.4.9.3460)
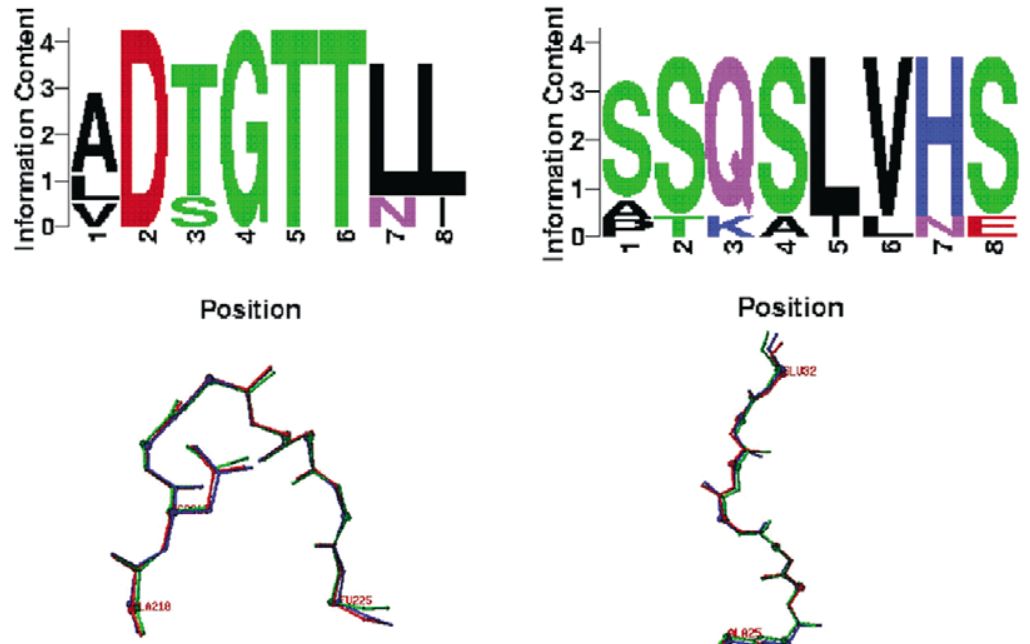

D. Acid Proteases: Active site loop conformation IV (F.b.50.1.4.10.12872)

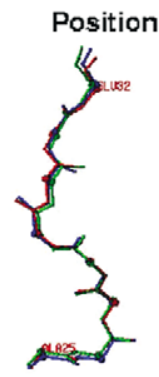

E. Immunoglobulin: Loop L1 (F.b.1.1.4.17.7805)

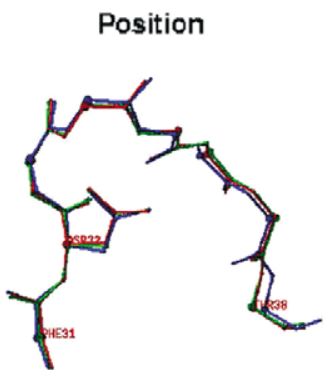

C. Acid Proteases: Active site loop conformation III (F.b.50.1.4.10.10386)

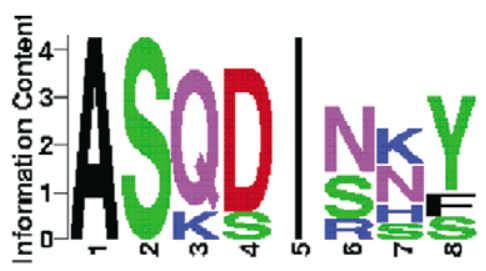

Position

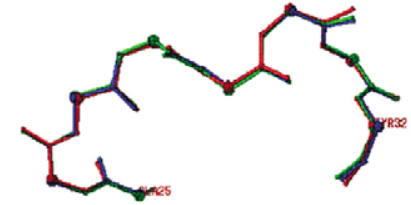

F. Immunoglobulin: Loop L1 (F.b.1.1.4.11.10249)

Figure 7. Sequence logos and superposed images for some representative functional clusters. Superposed images were generated using Swiss-PdbViewer ${ }^{30}$ and Sequence logos were generated as described. ${ }^{27}$ A, Acid proteases: active-site loop conformation I (F.b.50.1.3.11.7870) (1IDA-A-24,1C6Y-A-24,4FIV-A-29,0.2,0.2); B, acid proteases: activesite loop conformation II (F.b.50.1.4.9.3460) (1QDM-A-35,LYB-A-32, 1BXO-A-32,0.3,0.3); C, acid proteases: active-site loop conformation III (F.b.50.1.4.10.10386) (1MPP-A-31,2ASI-A-37, 1AM5-A-31,0.1,0.3); D, acid proteases: active-site loop conformation IV (F.b.50.1.4.10.12872) (1GVU-A-218,1FKN-A-227,1IBQ-A-213,0.2,0.2); E, immunoglobulin: loop L1 (F.b.1.1.4.17.7805) (1GPO-L-25,1LMK-A-225,1NQB-A-145,0.3,0.3); F, immunoglobulin: loop L1 (F.b.1.1.4.11.10249) (1FGN-L-25,1FGV-L-25,JFQ-L-25,0.2,0.1).

known $\beta$-hairpin (S.1.6.1.6.19) (1TGS-I-23, 1VMO-A-108,3IL8-A-41,0.3,0.4); E, known $\alpha$-turn- $\alpha$ (S.17.3.1.10.147) (1HUWA-67, 1VIN-A-347,1FXK-A-45,0.5,0.4); F, known $\beta$-turn- $\alpha$ (S.21.2.2..1083) (1LDM-A-24, 1GV0-A-4,3LAD-A-184,0.2,0.3); $\mathrm{G}$, clusters of peptides that have been assigned different class numbers but similar secondary structure flanks in the Loop database (S.14.1.11.363) (1NBA-A-220,3DFR-A-39,1I12-A-133,0.5,0.4); H, clusters of peptides that have been assigned different class numbers as well as secondary structure flanks in the Loop database (S.11.2.1.11.51) (1TGS-I25,1FBA-A-178,1TCA-A-57,0.5,0.6); I, new $\alpha$-turn- $\alpha$ (S.3.4.1.13.210) (1FO4-A-1053, 1QR0-A-58,1FO4-A-779,0.4,0.2); J, new $\alpha$-turn- $\alpha$ (S.17.6.1.10.264) (1QGR-A-702, 1BE3-F-47,1JB7-B-28,0.7,0.5); K, new $\beta$-hairpin (S.21.1.1.7.137) (2DPM-A135, 1DGJ-A-378,1TFP-A-35,0.5,0.5); L, new $\beta$-turn- $\alpha$ (S.3.1.1.15.559) (1RFN-A-121, 1F1C-A-18,1GC7-A-225,0.8,0.6). 
F.b.50.1.4.10.10386 and F.b.50.1.4.10.12872, contained non-retroviral proteins, while the fourth cluster, F.b.50.1.3.11.7870, contained retroviral proteins (Table 5). Each of the three non-retroviral clusters contained peptides of animal, plant as well as microbial proteins. Clusters F.b.50.1.4.9.346 and F.b.50.1.4.10.10386 were dominated by the peptides containing the first of the two catalytic aspartate residues, while the cluster F.b.50.1.4.10.12872 contained the second catalytic aspartate residue. Sequence profiles of all four clusters match with the PROSITE pattern of ASP_POTEASE, although there are noticeable differences in their sequence logos (Figure 7D). This highlights the differences in the loop conformation for the two loops that house the two catalytic aspartate residues in non-retroviral protein and the differences in catalytic loop conformation between the retroviral and non-retroviral proteins. This differentiation is not possible based on amino acid sequences alone as all the aspartic proteases satisfy the PROSITE sequence pattern of ASP_PROTEASE.

\section{Cysteine proteases}

Three of the functional clusters specific for cysteine proteases covered the three loop regions with each loop housing a catalytic residue from the Cys/His/Asn catalytic triad (Table 5). Cluster F.d.3.1.4.10.3457 houses the conserved Gln19 (1f2a numbering) in addition to the catalytic Cys25. Cluster F.d.3.1.3.18.4855 houses the catalytic His, while the cluster F.d.3.1.3.9.2416 houses the conserved Trp181 in addition to the catalytic Asn179. The conserved Gln19 and Trp181 are known to play a role in the transition state stabilization of the negatively charged oxyanion. ${ }^{35,36}$ Sequence profiles of the clusters F.d.3.1.4.10.3457, F.d.3.1.3.18.4855 and F.d.3.1.3.9.2416 match with the PROSITE sequence signatures of the cysteine proteases, THIOL_PROTEASE_CYS, THIOL_PROTEASE_HIS and THIOL_PROTEASE_ASN, respectively. Smaller size of a cluster that houses the catalytic His residue indicates the variable nature of the histidine-containing loop region compared to the loops that contain catalytic Asn and Cys. In addition, several functional clusters specific to cysteine proteases were detected within the loop regions.

\section{Trypsin-like serine protease}

The loops that house catalytic ${ }^{37,38}$ His57 and Asp102 take up at least two distinct conformations as indicated by two clusters for each region (Table 5). On the other hand, for the catalytic Ser195, a single cluster was detected, which accounts for 56 peptides from 56 proteins. Multiple clusters and smaller cluster sizes for clusters that house the catalytic His57 and Asp102 are indicative of the variable nature of these loops compared to what houses Ser195. This suggests that the loop that houses a catalytic residue can take up alternative conformations in different proteins yet maintain a conserved relative arrangement of the catalytic Ser/His/Asp residues across the family. Several other functional clusters were detected for this superfamily.

\section{EF-hand}

Several EF-hand specific functional clusters were detected that span the calcium-binding loop. ${ }^{39,40}$ Sequence profiles of these clusters match with the PROSITE sequence signature of EF-hand. For example, the largest cluster of this family, F.a.39.1.3.5.523, contains 63 peptides from 36 proteins (Table 5). This indicates the presence of multiple non-overlapping, superimposable peptides from a protein in a cluster. This is not surprising, as the presence of multiple calciumbinding loops per protein is a known phenomenon. ${ }^{39,40}$ Within these clusters, some of the conserved amino acid residue positions match with the reported residues that form the primary co-ordination sphere around the calcium ion.

\section{Immunoglobulin}

Immunoglobulin is an exceptional family, with its members gaining specificity with variation in loop regions on a relatively conserved framework. It is believed that the specificity is mainly determined by the sequence, size and, in turn, the structure of the hypervariable loop regions in the variable domains. ${ }^{15,16,41,42}$ Several "canonical structures" have been identified for the six hypervariable loop regions, three in the light chain and three in the heavy chain, by Chothia and co-workers. Martin \& Thornton ${ }^{14}$ have refined this classification via an automated clustering scheme. We find several functional clusters of peptides in the hypervariable loop regions (Table 5). We detect five clusters in the L1 region, four clusters in the L2 region, three clusters in the L3 region, three clusters in the $\mathrm{H} 1$ region, one cluster in the $\mathrm{H} 2$ region. The differences in the sequence profiles and conformation for two of the clusters corresponding to the $\mathrm{L} 1$ region are depicted in Figure 7E and F. No cluster was detected for the $\mathrm{H} 3$ region, indicating that the $\mathrm{H} 2$ and $\mathrm{H} 3$ regions are significantly more variable than the L1, L2, L3 and $\mathrm{H} 1$ regions. Our observation on the hypervariable nature of $\mathrm{H3}$ is in agreement with the observations by Chothia \& Lesk. ${ }^{15}$ The detected clusters correspond with the "canonical structures" described by Chothia et al. ${ }^{15}$ However, some of the canonical classes described by Chothia $^{15}$ or the classes described by Martin \& Thornton ${ }^{14}$ could not be detected in our clusters. This indicates that some of the loop regions of immunoglobulin are significantly unique in their structures and do not have five or more representatives of the conformation in the PDB_95 database, the minimum 
required to declare a cluster of conformations as dense. Several other functional clusters were observed for immunoglobulin, within the so-called conserved backbone regions of the proteins. This throws light on possible variations in the immunoglobulin structure outside the hypervariable loops.

\section{Discussion}

This is the first report of an objective classification of peptide structural fragments and provides a significant refinement over the conventional schemes of loop classification ${ }^{8,9}$ and secondary structure assignment based on dihederal angles or hydrogen bonding patterns. With the possibility of an infinite number of conformations that a peptide fragment can take up, it is interesting to note that $77 \%$ of the 1.2 million amino acid residues in 4849 PDB structures are described by 12,903 clusters or conformation types. These conformations are equivalent to the building blocks that nature uses while constructing a protein. Thus, we now describe the protein structure, a sequence of overlapping octapeptides, as a sequence of building blocks or conformation types. This study has significant implications in protein structure prediction as the proteins are likely to be made up of the building blocks described above.

The clusters were categorized as functional clusters if more than $70 \%$ of the peptides in a cluster are drawn from a single SCOP superfamily. ${ }^{31}$ This functional and structural categorization is useful in understanding whether the conformational conservation of the octapeptides in a cluster is a result of sequence homology amongst the proteins from which the peptides are drawn. The functional clusters provide clues about the potential functional sites of the protein families as described for the examples here. Of the 12,903 clusters, 10,696 are structural clusters, indicating that the conformation types are not merely a result of sequence homology or evolutionary relationship among proteins. The structural clusters are made up of regular secondary structures as well as loops. We discover several categories of $\alpha$-helices with differences in the regularity of the helix. Many of these different helix categories have been described qualitatively, ${ }^{10}$ but it has been difficult to assign these categories in an automated manner. Furthermore, it is well known that the beta strands can take up different geometrical structures and, therefore, two randomly chosen beta strand fragments are not necessarily superimposable. We discover many distinct clusters of beta strands where fragments belonging to a cluster are superimposable. Thus, beta strands cannot be considered as truly regular structures from a geometric point of view although they satisfy the loosely defined criteria of regularity of dihederal angles and hydrogen bond patterns.
We discover the majority of the categories of loops described before ${ }^{8,9,32}$ in our clusters. In addition, several hundred new loop categories are discovered. All of the reported loop classification schemes define loops as structures joining two regular secondary structural elements. The loop definition is thus fully dependent on the assignment of frontier amino acids between the secondary structure element and the loop. The length of the loop may vary depending on the secondary structure assignment program. ${ }^{8}$ The loop classification programs then cluster loops of identical length and joining similar type of secondary structure elements. This causes severe limitation to the methods, as the ambiguity in secondary structure assignment introduces a source of error. Our method overcomes this limitation by bringing all the possible octapeptide fragments, irrespective of secondary structure assignment, into one pool and then clustering them based on geometric similarity alone.

Clustering of the peptide fragment structures based on geometric invariants is a novel feature of this approach. Clustering of all the peptide fragments from the protein universe has not been reported before, possibly due to the enormous computational power required for superposing all possible pairs of fragments. We eliminate the pairwise comparison by describing the geometry of the fragment with a suite of geometric invariants. These invariants are based on the tetrahedra generated from the $\mathrm{C}^{\alpha}$ atoms of the fragment. Clustering of loops based on the phi, psi and omega torsion angles has been reported by Martin \& Thorton ${ }^{14}$ and by Oliva et al. ${ }^{9}$ These torsion angles are related to the volumes of the tetrahedra of the four atoms concerned. Kwasigroch et al. ${ }^{8}$ have suggested clustering of loops based on two distance measures: distance between the start (L1) and end (Ln) of the loop and the distance between the L1-Ln axis and the center of gravity of the loop backbone. In our analysis, we use a far greater number of invariants than used by Martin \& Thornton ${ }^{14}$ or Oliva et al. ${ }^{9}$ As we have described, ${ }^{23}$ volume is a third-order invariant. In addition, we use several first-order (length related) and second-order (area related) invariants to describe a peptide fragment. Use of geometric invariants of different order and therefore different degree of sensitivity ${ }^{25}$ provides an advantage.

Our algorithm does not require assignment of secondary structure or classification based on the regions of the Ramachandran plot. We have shown that proteins can be considered merely as geometric objects and subjected to clustering. This also shows that the understanding of secondary structures and hydrogen bonding patterns is not essential while classifying peptide structure fragments.

It may be noted that our results are based on certain adjustable parameters in our algorithm. These parameters include the maximum allowable dimensions of the cluster, $W_{i}$, in each of the 56 
dimensions and the minimum number of peptides in a cluster before the cluster is declared as a dense cluster. We have obtained our results with the parameters shown in Table 1, which have been obtained after extensive training on known similar fragments. Thus, it is possible to further refine the results by variation of the parameters. For example, the dimensions of the cluster may be relaxed to capture a larger number of peptides in a cluster as well as a larger number of clusters. Furthermore, the length of the peptide used in clustering is likely to have significant impact on the results. We have chosen octapeptides, which enables us to capture the majority of the secondary structures as well as the loops with at least one flanking secondary structure.

As a large number of conformation types are covered in our database of clusters, it is possible to generate structure-sequence relationship from the sequence conservation patterns in the clusters. This is different from the work by Baker and co-workers, ${ }^{12,43}$ as our clusters have originated from structural similarities rather than sequence similarities. We propose that the sequence profiles of these clusters can be used to model structures of new proteins based on our fragment library.

\section{Acknowledgements}

The work was funded, in part, by a grant from the Council of Scientific and Industrial Research, Government of India. The authors acknowledge useful discussions with Sunita Sarawagi \& Sachin Patkar of Indian Institute of Technology, Bombay on the clustering strategy.

\section{References}

1. Sibanda, B. L., Blundell, T. L. \& Thornton, J. M. (1989). Conformation of $\beta$-hairpins in protein structures. A systematic classification with applications to modeling by homology, electron density fitting and protein engineering. J. Mol. Biol. 206, 759-777.

2. Leszczynski, J. F. \& Rose, G. D. (1986). Loops in globular proteins: a novel category of secondary structure. Science, 234, 849-855.

3. Sibanda, B. L. \& Thornton, J. M. (1985). $\beta$-Hairpin families in globular proteins. Nature, 316, 170-175.

4. Milner-White, E. J. \& Poet, R. (1987). Loops, bulges, turns and hairpins in proteins. Trends Biochem. Sci. 12, 189-192.

5. Milner-White, E. J. (1986). Classification of $\beta$-hairpin turns. Biochem. Soc. Trans. 14, 877.

6. Milner-White, E. J. \& Poet, R. (1986). Four classes of $\beta$-hairpins in proteins. Biochem. J. 240, 289-292.

7. Wintjens, R., Rooman, M. J. \& Wodak, S. J. (1996). Automatic classification and analysis of $\alpha \alpha$-turn motifs in proteins. J. Mol. Biol. 255, 235-253.

8. Kwasigroch, J.-M., Chomilier, J. \& Mornon, J.-P. (1996). A global taxonomy of loops in globular proteins. J. Mol. Biol. 259, 855-872.

9. Oliva, B., Bates, P. A., Querol, E., Aviles, F. X. \&
Sternberg, M. J. E. (1997). An automated classification of the structure of protein loops. J. Mol. Biol. 266, 814-830.

10. Richardson, J. S. (1981). The anatomy and taxonomy of protein structure. Advan. Protein Chem. 34, 167-339.

11. Martin, A. C. R., Toda, K., Stirk, H. \& Thornton, J. M. (1995). Long loops in proteins. Protein Eng. 8, 1093-1101.

12. Bystroff, C. \& Baker, D. (1998). Prediction of local structure in proteins using a library of sequencestructure motifs. J. Mol. Biol. 281, 565-577.

13. Bystroff, C., Thorsson, V. \& Baker, D. (2000). HMMSTR: a hidden Markov model for local sequence-structure correlations in proteins. J. Mol. Biol. 301, 173-190.

14. Martin, A. C. R. \& Thornton, J. M. (1996). Structural families in loops of homologous proteins: automatic classification, modeling and application to antibodies. J. Mol. Biol. 263, 800-815.

15. Chothia, C. \& Lesk, A. M. (1987). Canonical structures for the hypervariable regions of immunoglobulins. J. Mol. Biol. 196, 901-917.

16. Chothia, C., Lesk, A. M., Gherardi, E., Tomlinson, I. M., Walter, G., Marks, J. D. et al. (1992). Structural repertoire of the human VH segments. J. Mol. Biol. 227, 799-817.

17. Saqi, M. A. \& Sternberg, M. J. E. (1994). Identification of sequence motifs from a set of proteins with related function. Protein Eng. 7, 165-171.

18. Bairoch, A., Bucher, P. \& Hofmann, K. (1997). The PROSITE database, its status in 1997. Nucl. Acids Res. 25, 217-221.

19. Attwood, T. K., Beck, M. E., Bleasby, A. J. \& ParrySmith, D. J. (1994). PRINTS-a database of protein motif fingerprints. Nucl. Acids Res. 22, 3590-3596.

20. Henikoff, S., Pietrokovski, S. \& Henikoff, J. G. (1998). Superior performance in protein homology detection with the Blocks Database servers. Nucl. Acids Res. 26, 309-312.

21. Kasuya, A. \& Thornton, J. M. (1999). Threedimensional structure analysis of PROSITE patterns. J. Mol. Biol. 286, 1673-1691.

22. Brenner, S. E., Koehl, P. \& Levitt, M. (2000). The ASTRAL compendium for protein structure and sequence analysis. Nucl. Acids Res. 28, 254-256.

23. Tendulkar, A. V., Wangikar, P. P., Sohoni, M. A., Samant, V. V. \& Mone, C. Y. (2003). Parameterization and classification of the protein universe via geometric techniques. J. Mol. Biol. 334, 157-172.

24. Hilbert, D. (1983). Uber die vollen Invariantensysteme. Math. Ann. 42, 313-373.

25. Weyl, H. (1939). The Classical Groups, their Invariants and Representations, Princeton University Press, Princeton.

26. Mumford, D., Fogarty, J. \& Kirwan, F. (1994). Geometric Invariant Theory, Springer, New York.

27. Schneider, T. D. \& Stephens, R. M. (1990). Sequence logos: a new way to display consensus sequences. Nucl. Acids Res. 18, 6097-6100.

28. Berman, H. M., Westbrook, J., Feng, Z., Gilliland, G., Bhat, T. N., Weissig, H. et al. (2000). The Protein Data Bank. Nucl. Acids Res. 28, 235-242.

29. Bernstein, F. C., Koetzle, T. F., Williams, G. J. B., Meyer, E. F., Brice, M. D., Rodgers, J. R. et al. (1977) The Protein Data Bank: a computer-based archival file for macromolecular structures. J. Mol. Biol. 122, $535-542$.

30. Guex, N. \& Peitsch, M. C. (1997). SWISS-MODEL 
and the Swiss-PdbViewer: an environment for comparative protein modeling. Electrophoresis, 18, 2714-2723.

31. Murzin, A. G., Brenner, S. E., Hubbard, T. \& Chothia, C. (1995). SCOP: a structural classification of proteins database for the investigation of sequences and structures. J. Mol. Biol. 247, 536-539.

32. Burke, D. F., Deane, C. M. \& Blundell, T. L. (2000). Browsing the SLoop database of structurally classified loops connecting elements of protein secondary structure. Bioinformatics, 16, 513-519.

33. Bairoch, A. (1993). The PROSITE dictionary of sites and patterns in proteins, its current status. $\mathrm{Nucl}$. Acids Res. 21, 3097-3103.

34. Newman, M., Watson, F., Roychowdhury, P., Jones, H., Badasso, M., Cleasby, A. et al. (1993). X-ray analysis of aspartic proteinases $\mathrm{V}$ : structure and refinement at $2.0 \AA$ resolution of the aspartic proteinase from Mucor pusillus. J. Mol. Biol. 230, 260-283.

35. Murthy, S. N. P., Iismaa, S., Begg, G., Freymann, D. M., Graham, R. M. \& Lorand, L. (2002). Conserved tryptophan in the core domain of transglutaminase is essential for catalytic activity. Proc. Natl Acad. Sci. USA, 2002, 2738-2742.

36. Menard, R., Carriere, J., Laflamme, P., Plouffe, C., Khouri, H. E., Vernet, T. et al. (1991). Contribution of the glutamine 19 side chain to transition-state stabilization in the oxyanion hole of papain. Biochemistry, 30, 8924-8928.

37. Blow, D. M. (1997). The tortuous story of Asp..His...Ser: structural analysis of small alpha, Greek-chymotrypsin. Trends Biochem. Sci. 22, 405-408.

38. Carter, P. \& Wells, J. (1988). Dissecting the catalytic triad of a serine protease. Nature, 332, 564-568.

39. Swain, A. L., Kretsingerl, R. H. \& Amma, E. L. (1989). Restrained least squares refinement of native (calcium) and cadmium-substituted carp parval- bumin using X-ray crystallographic data at 1.6resolution. J. Biol. Chem. 264, 16620-16628.

40. Ames, J. B., Dizhoor, A. M., Ikura, M., Palczewski, K. \& Stryerii, L. (1999). Three-dimensional structure of guanylyl cyclase activating protein-2, a calciumsensitive modulator of photoreceptor guanylyl cyclases. J. Biol. Chem. 274, 19329-19337.

41. Chothia, C., Lesk, A. M., Levitt, M., Amit, A. G., Mariuzza, R. A., Phillips, S. E. V. \& Poljak, R. J. (1986). The predicted structure of immunoglobulin D1.3 and its comparison with the crystal structure. Science, 233, 755-758.

42. Chothia, C., Lesk, A. M., Tramontano, A., Levitt, M., Smith-Gill, S. J., Air, G. et al. (1989). Conformations of immunoglobulin hypervariable regions. Nature, $342,877-883$.

43. Baker, D. \& Sali, A. (2001). Protein structure prediction and structural genomics. Science, 294, 93-96. 